\title{
Faster Matroid Intersection
}

\author{
Deeparnab Chakrabarty* $\quad$ Yin Tat Lee $^{\dagger} \quad$ Aaron Sidford $^{\ddagger} \quad$ Sahil Singla $^{\S}$ \\ Sam Chiu-wai Wong
}

November 26, 2019

\begin{abstract}
In this paper we consider the classic matroid intersection problem: given two matroids $\mathcal{M}_{1}=\left(V, \mathcal{I}_{1}\right)$ and $\mathcal{M}_{2}=\left(V, \mathcal{I}_{2}\right)$ defined over a common ground set $V$, compute a set $S \in \mathcal{I}_{1} \cap \mathcal{I}_{2}$ of largest possible cardinality, denoted by $r$. We consider this problem both in the setting where each $\mathcal{M}_{i}$ is accessed through an independence oracle, i.e. a routine which returns whether or not a set $S \in \mathcal{I}_{i}$ in $\mathcal{T}_{\text {ind }}$ time, and the setting where each $\mathcal{M}_{i}$ is accessed through a rank oracle, i.e. a routine which returns the size of the largest independent subset of $S$ in $\mathcal{M}_{i}$ in $\mathcal{T}_{\text {rank }}$ time.

In each setting we provide faster exact and approximate algorithms. Given an independence oracle, we provide an exact $O\left(n r \log r \cdot \mathcal{T}_{\text {ind }}\right)$ time algorithm. This improves upon previous best known running times of $O\left(n r^{1.5} \cdot \mathcal{T}_{\text {ind }}\right)$ due to Cunningham in $1986[6]$ and $\tilde{O}\left(n^{2} \cdot \mathcal{T}_{\text {ind }}+n^{3}\right)$ due to Lee, Sidford, and Wong in 2015 [27]. We also provide two algorithms which compute a $(1-\epsilon)$-approximate solution to matroid intersection running in times $\tilde{O}\left(n^{1.5} / \epsilon^{1.5} \cdot \mathcal{T}_{\text {ind }}\right)$ and $\tilde{O}\left(\left(n^{2} r^{-1} \epsilon^{-2}+r^{1.5} \epsilon^{-4.5}\right) \cdot \mathcal{T}_{\text {ind }}\right)$, respectively. These results improve upon the $O\left(n r / \epsilon \cdot \mathcal{T}_{\text {ind }}\right)$-time algorithm of Cunningham [6] as noted recently by Chekuri and Quanrud [3].

Given a rank oracle, we provide algorithms with even better dependence on $n$ and $r$. We provide an $O\left(n \sqrt{r} \log n \cdot \mathcal{T}_{\text {rank }}\right)$-time exact algorithm and an $O\left(n \epsilon^{-1} \log n \cdot \mathcal{T}_{\text {rank }}\right)$-time algorithm which obtains a $(1-\epsilon)$-approximation to the matroid intersection problem. The former result improves over the $\tilde{O}\left(n r \cdot \mathcal{T}_{\text {rank }}+n^{3}\right)$-time algorithm by Lee, Sidford, and Wong [27]. The rank oracle is of particular interest as the matroid intersection problem with this oracle is a special case (via Edmond's minimax characterization of matroid intersection) of the submodular function minimization (SFM) problem with an evaluation oracle, and understanding SFM query complexity is an outstanding open question.
\end{abstract}

*Dartmouth College, deeparnab@dartmouth.edu

${ }^{\dagger}$ University of Washington and Microsoft Research, yintat@uw.edu

${ }^{\ddagger}$ Stanford University, sidford@stanford.edu. Research supported in part by NSF CAREER Award CCF-1844855.

§Princeton University and Institute for Advanced Study, singla@cs.princeton.edu

`Microsoft Research, samwon@microsoft.com 


\section{Contents}

1 Introduction $\quad 3$

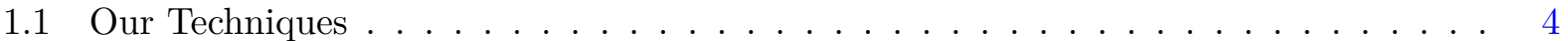

1.2 Related Works . . . . . . . . . . . . . . . . . . . 6

2 Preliminaries $\quad 7$

2.1 Notation . . . . . . . . . . . . . . . . . . . 7

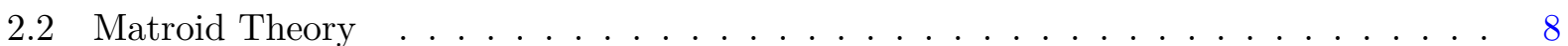

3 Exchange Graph Exploration via Binary Search $\quad 9$

3.1 Finding Free Vertices using Rank Oracle . . . . . . . . . . . . . . . . . . . . 10

3.2 Finding Exchange Vertices using Independence or Rank Oracle . . . . . . . . . . . . 10

4 Exact and Approximation Algorithms using Rank Oracle 11

4.1 Exploring the Exchange Graph with a Rank Oracle . . . . . . . . . . . . . . . 12

4.2 Blocking Flow with Rank Oracle . . . . . . . . . . . . . . . . . . 13

4.3 Matroid Intersection with a Rank Oracle . . . . . . . . . . . . . . . . . . 15

5 Exact Algorithm using Independence Oracle $\quad 16$

5.1 Finding the Distances . . . . . . . . . . . . . . . . . . 16

5.2 Finding One Augmenting Path . . . . . . . . . . . . . . . . . . 18

5.3 The Augmenting Paths Algorithm . . . . . . . . . . . . . . . . . . . 19

6 Approximation Algorithm using Independence Oracle 20

6.1 Facts about Matroids . . . . . . . . . . . . . . . . . . . . . . . 20

6.2 Augmenting Sets . . . . . . . . . . . . . . . . . . . . 21

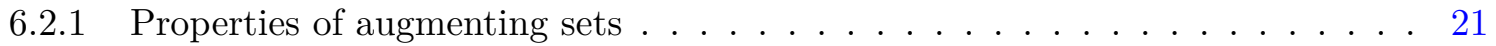

6.2.2 Partial Augmenting Sets . . . . . . . . . . . . . . . . 25

6.3 The Augmenting Sets Algorithm . . . . . . . . . . . . . . . . . 27

6.3.1 An Analogy and High level idea . . . . . . . . . . . . . . . . 27

6.3 .2 The Detailed Algorithm . . . . . . . . . . . . . . . . . . 27

6.4 Going Subquadratic by Combining Augmenting Sets and Augmenting Paths . . . . . 31

7 Approximately Optimal Fractional Solution via Frank-Wolfe 32

8 Faster Algorithms by Sparsification $\quad 34$ 


\section{Introduction}

A matroid $\mathcal{M}=(V, \mathcal{I})$ is an abstract set system defined over a finite ground set (universe) $V$ of size $n$ where the collection $\mathcal{I} \subseteq 2^{V}$ of independent sets satisfy two properties: (a) $A \in \mathcal{I}$ implies every subset $B \subseteq A$ is also independent, i.e., $B \in \mathcal{I}$, and (b) for any two sets $A, B \in \mathcal{I}$ with $|A|<|B|$, there exists an element $e \in B \backslash A$ such that $A+e \in \mathcal{I}$. Matroids are fundamental objects in combinatorics, and the abstract definition above generalizes a wide range of concepts ranging from acyclic graphs to linearly independent matrices.

Given two matroids $\mathcal{M}_{1}=\left(V, \mathcal{I}_{1}\right)$ and $\mathcal{M}_{2}=\left(V, \mathcal{I}_{2}\right)$ over the same ground set, the matroid intersection problem is to find a set $I \in \mathcal{I}_{1} \cap \mathcal{I}_{2}$ with the largest cardinality $|I|$. This problem generalizes many important combinatorial optimization problems such as bipartite matching, arborescences in digraphs, and packing spanning trees. Unsurprisingly, this problem has applications in areas such as electrical engineering [30, 33] and network coding [7, 10, 8].

To define the problem algorithmically, one needs to specify access to these matroids. In the literature it is common to assume access to an independence oracle which takes as input a subset $S \subseteq V$ and returns whether $S \in \mathcal{I}$ or not. We use $\mathcal{T}_{\text {ind }}$ to denote the maximum time taken by such an oracle to answer a single query. This raises an algorithmic question: in how few queries can the matroid intersection problem be solved?

Starting from the work of Edmonds [9], many polynomial time algorithms [1, 25, 6, 13, 36, 27] have been proposed for the matroid intersection problem. The previous state-of-the-art captured by two works. One is a classic $O\left(n r^{1.5} \cdot \mathcal{T}_{\text {ind }}\right)$ time combinatorial algorithm by Cunningham [6] where $r$ is the cardinality of the largest common independent set of the two matroids. The second algorithm is an $\tilde{O}\left(n^{2} \cdot \mathcal{T}_{\text {ind }}+n^{3}\right)$-time algorithm by Lee, Sidford, and Wong [27] based on fast algorithms implementing the ellipsoid method. Note that this pays a higher overhead than Cunningham's result and makes many more queries if $r \ll n^{2 / 3}$. This raises the important question, "Is there an algorithm which obtains the best of both results?" Our first result answers this affirmatively (restated as Theorem 18 in the main body). ${ }^{1}$

Theorem 1. There is an $O\left(n r \log r \cdot \mathcal{T}_{\text {ind }}\right)$-time algorithm to solve the matroid intersection problem exactly.

Our next result looks at approximate matroid intersection. Due to both theoretical and practical reasons, there has been extensive recent work [5, 26, 34, 24, 31, 3, 21, 35, 37] in trying to obtain faster $(1 \pm \epsilon)$-approximation algorithms for problems which already have polynomial time exact algorithms. A $(1-\epsilon)$-approximate algorithm for matroid intersection would return a set $I \in \mathcal{I}_{1} \cap \mathcal{I}_{2}$ with $|I| \geq(1-\epsilon) r$ where $r$ is the cardinality of largest common independent set.

For matroid intersection, Cunningham's [6] algorithm already gives a $(1-\epsilon)$-approximate solution in $O\left(n r / \epsilon \cdot \mathcal{T}_{\text {ind }}\right)$ time. This observation was made explicit in a recent paper by Chekuri and Quanrud [3]. Given our exact algorithm result above, it is natural to wonder if one can obtain even faster approximation algorithms. In particular, can there be subquadratic approximation algorithms for the matroid intersection algorithm problem? Our second result gives an affirmative answer (restated as Theorem 21 later).

Theorem 2. There is an $O\left(n^{1.5} \sqrt{\log r} / \epsilon^{1.5} \cdot \mathcal{T}_{\text {ind }}\right)$-time algorithm to obtain a $(1-\epsilon)$-approximation to the matroid intersection problem.

\footnotetext{
${ }^{1}$ In simultaneous and independent work [11], Nguyễn also answered this question affirmatively obtaining an $O\left(n r(\log r)^{2} \cdot \mathcal{T}_{\text {ind }}\right)$-time algorithm for solving the matroid intersection problem exactly. See the remark at the end of Section 5 for a comparison.
} 
Theorem 2 has no significant dependence on $r$, and indeed when $r \ll \sqrt{n}$, as it stands, it is better to use the exact algorithm. However, we can get a better result (restated as Theorem 55 in the main body) in the regime $\sqrt{n} \ll r \ll n$; concretely, assume $r=\Theta\left(n^{c}\right)$ where $\frac{1}{2}<c<1$.

Theorem 3. There is an $\tilde{O}\left(\left(\frac{n^{2}}{r \epsilon^{2}}+\frac{r^{1.5}}{\epsilon^{4.5}}\right) \cdot \mathcal{T}_{\text {ind }}\right)$-time algorithm to obtain a $(1-\epsilon)$-approximation to the matroid intersection problem.

The Rank Oracle. Another common model for accessing matroids is a rank oracle. Given a subset $S \subseteq V$, the rank oracle outputs $\operatorname{rank}(S)$, i.e., the size of the maximum cardinality independent subset of $S$. We let $\mathcal{T}_{\text {rank }}$ denote the maximum time taken by the rank oracle to answer any query. The rank oracle is clearly at least as powerful as the independence oracle.

Similar to the independence oracle, Lee, Sidford, and Wong [27] also gave an algorithm for the rank oracle with a runtime of $\tilde{O}\left(n r \cdot \mathcal{T}_{\text {rank }}+n^{3}\right)$. This suggests that perhaps matroid intersection can be solved strictly faster in the rank oracle model.

One reason to look at the rank oracle is Edmonds' minimax theorem [9] which states that $\max _{I \in \mathcal{I}_{1} \cap \mathcal{I}_{2}}|I|=\min _{S \subseteq V}\left(\operatorname{rank}_{1}(S)+\operatorname{rank}_{2}(V \backslash S)\right)$. Since the rank function is submodular, (the dual of) matroid intersection is a special case of submodular function minimization (SFM) where the function evaluation oracle corresponds to (two calls of) the rank oracle. SFM is an extensively studied problem whose query complexity is still an open question. In this light, understanding it for the special case of matroid intersection becomes an important problem.

Another reason is that our main ideas for designing independence oracle-based algorithms arose from an understanding the question with rank oracle, and so the rank oracle formulation constitutes a natural warm-up. In this paper we provide the following results on solving matroid intersection (both approximately and exactly with a rank oracle), improving over the $\tilde{O}\left(n r \cdot \mathcal{T}_{\text {rank }}+n^{3}\right)$-time algorithm by Lee, Sidford, and Wong [27] (restated as Theorem 16 and Theorem 17 later).

Theorem 4. There is an $O\left(n \sqrt{r} \log n \cdot \mathcal{T}_{\text {rank }}\right)$-time algorithm to exactly solve the matroid intersection problem. There is an $O\left(n \epsilon^{-1} \log n \cdot \mathcal{T}_{\text {rank }}\right)$-time algorithm to obtain a $(1-\epsilon)$-approximation to the matroid intersection problem.

\subsection{Our Techniques}

At a high level our algorithms build upon existing combinatorial algorithms, i.e. Edmonds [9] and Cunningham [6]. We extend these algorithms leveraging the following key ideas: the binary search idea which allows fast exploration through the exchange graph, the augmenting sets methodology which allows multiple parallel augmentations in the exchange graph, and first-order methods which allow efficient sparsification of the ground set. In this section we explain each idea in greater depth, leaving the full details to subsequent sections. We begin with a refresher and a high-level overview of existing combinatorial algorithms.

The exchange graph and the algorithms of Edmonds and Cunningham. The key object behind almost all combinatorial algorithms for matroid intersection is the exchange graph. Given a current solution $S \in \mathcal{I}_{1} \cap \mathcal{I}_{2}$, the exchange graph $G(S)$ is a directed bipartite graph where the endpoints of arcs correspond to valid exchanges in respective matroids depending on the direction. There is a source $s$ and sink $t$. The source is connected to all vertices in $V \backslash S$ which can be freely added in one matroid, and the sink is connected from all vertices in $V \backslash S$ which can be freely added to the other matroid. Much as in network flow theory, an improvement in the size of $S$ arises on finding shortest augmenting source-sink paths in this exchange graph. At a very high 
level, there are $O(n r)$ possible edges in the graph (since $|S| \leq r$ ), each edge can be constructed with $O(1)$ independence oracle queries, and in at most $r$-augmentations one obtains the maximum sized common independent set. This gives an $O\left(n r^{2} \cdot \mathcal{T}_{\text {ind }}\right)$-time algorithm $[9,1,25]$.

Cunningham's [6] main idea was to implement the above algorithm in phases. This idea is similar to the Hopcroft-Karp [20] idea for bipartite matching but has many differences. Akin to [20], in each "early" phase the algorithm tries to find many disjoint short augmenting paths; however, not all of these can be augmented in parallel. Indeed, one of the major divergences between bipartite matching and matroid intersection is that even a single augmentation can completely change the exchange graph. Although the augmentations cannot be done in parallel, Cunningham [6] shows how to spend only $O(n r)$ independence-oracle calls (each edge is queried only once in a phase) to sequentially run all the augmentations in a single phase; as in Hopcroft-Karp [20], the early phases lead to big improvements and the total number of phases is at most $O(\sqrt{r})$. This leads to a total of $O\left(n r^{1.5} \cdot \mathcal{T}_{\text {ind }}\right)$-time algorithm.

The binary search idea. We start by describing the idea when we have a rank oracle and then discuss the independence oracle case. The first thing to note is that one doesn't need the whole exchange graph for an augmentation. Instead, what we need is to perform a breadth first search (BFS) on this "unknown" exchange graph and the rank oracle provides the following access to this graph: for every vertex $a$ and every subset $B \subseteq V \backslash v$, in $O(\log n)$ rank-oracle calls (by doing a binary-search style argument) we can detect if $a$ has an edge to some vertex in $B$ or not. This suffices for doing a BFS on the graph in $O\left(n \log n \cdot \mathcal{T}_{\text {rank }}\right)$ time, and we do it at the beginning of each phase of Cunningham's algorithm.

The second observation is that each phase in Cunningham's algorithm can be implemented using $O(n \log n)$-many rank-oracle calls again. Indeed, once the distances of vertices are known, shortest paths can be computed using the aforementioned graph access which the rank-oracle provides us. It is true that after some augmentations, some vertices will be misclassified (or will be useless, to borrow Cunningham's [6] terminology) but such vertices are never queried again. The latter requires some distance-monotonicity properties from [6]. Theorem 4 actually follows quite easily now: for an exact algorithm, there are $O(\sqrt{r})$ such phases; for a $(1-\epsilon)$-approximate algorithm, we need only $O\left(\frac{1}{\epsilon}\right)$-phases. Details of this are given in Section 4 .

Things are a bit trickier using only an independence oracle, since we cannot detect, in $O(\log n)$ queries, whether a vertex $a$ has an edge to a subset $B$ or not. Nevertheless, the following is true: for a vertex $a \notin S$ (recall $S$ is the current solution) and a subset $B \subseteq S$, in $O(\log r)$ independence queries we can figure out whether $a$ has an edge to/from a vertex in $B$. This is due to the way the exchange graph is defined. Armed with this observation, after every augmentation, we can perform a BFS of the new exchange graph (i.e., find the distance labels of all vertices) in $O(n \log r)$ independence oracle calls plus an $\tilde{O}(1)$ "amortized" independence call per vertex whose distance label changes in the current iteration. Since each vertex changes its distance label at most $r$ times, the total amortized cost is $O\left(n r \cdot \mathcal{T}_{\text {ind }}\right)$. Since there are at most $r$ augmentations, the total time taken in the (non-amortized part of the) BFS computations is $O\left(n r \log r \cdot \mathcal{T}_{\text {ind }}\right)$. Details of this are described in Section 5.

Augmenting sets. Our exact algorithm with independence oracle queries has two kinds of cost, both of which are $\tilde{O}\left(n r \cdot \mathcal{T}_{\text {ind }}\right)$-time. The "amortized" cost which is paid per "distance increase" per vertex can be made $\tilde{O}(n / \epsilon)$ if we run only $1 / \epsilon$ phases. However, the "non-amortized" cost is paid per augmentation, and this can still be $\tilde{O}(n r)$ even with $1 / \epsilon$ phases. Indeed, the phases don't seem to add any advantage. It seems unlikely that the binary search idea alone can be salvaged to 
break the "quadratic barrier" (when $r \approx n$ ).

To overcome this and prove Theorem 2, we propose the idea of augmenting sets. Recall the idea presented in Cunningham's algorithm: the algorithm is akin to Hopcroft-Karp [20] in that it runs in phases, and in each phase it augments along multiple augmenting paths. However, the big difference is that the augmentations need to be performed sequentially rather than in parallel as in Hopcroft-Karp, and this takes time. On the other hand, if we could find all the augmentations that can occur up front, then we will save on the time taken to find them sequentially. This is what our notion of augmenting sets achieves.

An augmenting set is a sequence of disjoint subsets $B_{1}, A_{1}, B_{2}, \ldots, A_{\ell}, B_{\ell+1}$ where alternate subsets are not in, and respectively in, the current solution $S$. More precisely, the set $A_{i}$ is a subset of vertices at distance exactly $2 i$ from the source of the exchange graph, and vertices $B_{i}$ is at distance $2 i-1$ from the source. Each subset has the same cardinality and finally, deleting all the $A_{i}$ 's and adding all the $B_{i}$ 's preserves independence in both matroids. If the size of each set is 1 , an augmenting set is the same as an augmenting path.

We prove an equivalence between augmenting sets and a collection of augmenting paths which can be augmented sequentially in the exchange graph. We introduce the concept of maximal augmenting sets, and show (a) as long as the shortest path is small, say $\ell$ (early phases of the algorithm), the maximal and maximum augmenting sets are within a multiplicative $O(\ell)$-factor, and (b) we show an algorithm to find a "near maximal" augmenting set, which allows us to guarantee that in any phase, and for any $p$, after spending $O\left(n^{2} / p \cdot \mathcal{T}_{\text {ind }}\right)$ time the maximum number of remaining $\ell$-length augmentations is $O(p \ell)$. These final augmentations can be done in $\tilde{O}\left(n p \ell \cdot \mathcal{T}_{\text {ind }}\right)$ time using the previous binary search ideas. Setting $p \approx \sqrt{n}$ gives the desired result (Theorem 2 ). The precise definition of augmenting sets, its properties, and the details of above ideas are in Section 6. We are hopeful that this new class of algorithms, which may be of independent interest, find further applications in related problems.

Frank-Wolfe sparsification and sampling. To obtain Theorem 3, we need one additional idea. Since we are being approximate, we can further improve the running time of our algorithm by sparsifying the ground set from $n$ to $\tilde{O}\left(r / \epsilon^{2}\right)$. We first look at the fractional solution to the matroid intersection problem as a convex optimization problem. Next, we observe that if we apply a constrained first-order method (aka the Frank-Wolfe algorithm), each step of the algorithm corresponds to solving the single matroid optimization problem which can be done by the greedy algorithm in $O\left(n \log n+n \cdot \mathcal{T}_{\text {ind }}\right)$ time. Furthermore, to get an $\epsilon$-optimal fractional solution, one needs only $\tilde{O}\left(n / r \epsilon^{2}\right)$ iterations which takes $\tilde{O}\left(\frac{n^{2}}{r \epsilon^{2}} \cdot \mathcal{T}_{\text {ind }}\right)$ time.

Next, we apply a sparsification procedure due to Karger [23] that leads us to a ground set with only $\tilde{O}\left(r / \epsilon^{2}\right)$ elements (instead of $n$ elements), and which has a $(1-O(\epsilon))$-approximate common independent set. Once the ground set shrinks, we can apply the algorithm from the previous section (using augmenting sets) to get a $(1-\epsilon)$-approximate solution in $\tilde{O}\left(r^{1.5} / \epsilon^{4.5} \cdot \mathcal{T}_{\text {ind }}\right)$ time. Taking everything together gives us Theorem 3. The details are given in Section 8 .

\subsection{Related Works}

Polynomial time algorithms for matroid intersection are more than forty years old, with the first algorithms present in the works of $[9,1,25]$. The running time of these algorithms were $O\left(n r^{2} \cdot \mathcal{T}_{\text {ind }}\right)$. Indeed, many of these papers $[25,12,2,13,14,36,27,21]$ looked at the weighted matroid intersection problem, and gave polynomial time algorithms.

For certain special matroids faster algorithms are known. Indeed, when the matroid is given explicitly, one can talk of pure running time instead of oracle queries. For instance, for exact 
maximum cardinality bipartite matching, the best running time is the $O(m \sqrt{n})$-time algorithm due to [20] and $\tilde{O}\left(m^{10 / 7}\right)$-time algorithm due to Macdry [28]. Here $m$ is the number of edges in the graph (and so, the number of elements in the matroid), while $n$ is the number of vertices which is the rank of the matroid. It is instructive to compare what our results give: we give an $\tilde{O}\left(m \sqrt{n} \cdot \mathcal{T}_{\text {rank }}\right)$ and $\tilde{O}\left(m n \cdot \mathcal{T}_{\text {ind }}\right)$ time algorithm. In dense graphs, the best algorithm is an $O\left(n^{\omega}\right)$ running time algorithm by $[29,17]$, where $\omega$ is the exponent of matrix multiplication. For linear matroids, the matroid intersection problem can be solved in essentially $O\left(n r^{\omega-1}\right)$-time $[17,4]$. For graphic matroids, the matroid intersection problem can be solved in $O(n \sqrt{r} \log r)$ time $[13,14]$.

The study of approximate matroid intersection problems is newer. As Chekuri and Quanrud [3] note, Cunningham's analysis implies a $O\left(n r / \epsilon \cdot \mathcal{T}_{\text {ind }}\right)$-time algorithm to get an $(1-\epsilon)$-approximate matroid intersection. Huang et al. [21] and Chekuri and Quanrud [3] study the approximate weighted version. The former gives an $\tilde{O}\left(n r^{1.5} / \epsilon \cdot \mathcal{T}_{\text {ind }}\right)$-time approximation algorithm [21], while the latter gives an $O\left(n r \log ^{2}(1 / \epsilon) / \epsilon^{2} \cdot \mathcal{T}_{\text {ind }}\right)$-time approximation algorithm. Contrast this with our $\tilde{O}\left(n r \cdot \mathcal{T}_{\text {ind }}\right)$-time exact and $\tilde{O}\left(n^{1.5} / \epsilon^{1.5}\right)$-time approximate algorithm, albeit for the unweighted version. Finally, Guruganesh and Singla [15] give an $\frac{1}{2}+\delta$-approximation algorithm for a small but fixed constant $\delta$ which runs in $O\left(n \cdot \mathcal{T}_{\text {ind }}\right)$-time.

We end the introduction with (to our knowledge) the only known lower bound for matroid intersection. Since we are in the oracle (rank/independence) model, it is perhaps foreseeable that some non-trivial information theoretic lower bound can be attained for the number of queries required for matroid intersection. Unfortunately, the only lower bound we are aware of is due to Harvey [18, 16]. For matroids with $r=n / 2$, Harvey [18] shows a lower bound of $\left(\log _{2} 3\right) n-o(n)$ queries. Obtaining an $\omega(n)$ lower bound is a challenging open problem.

\section{Preliminaries}

Here we provide the notation (Section 2.1) and previous known results about the matroid intersection (Section 2.2) that we use throughout the paper.

\subsection{Notation}

Here we provide the notational conventions we use throughout the paper.

Set Notation: We often work with subsets of a finite set $V$ which we call the universe or ground set. For $I \subseteq V$ and $a \in V$ we let $I+a \stackrel{\text { def }}{=} I \cup\{a\}$ and $I-a \stackrel{\text { def }}{=} I \backslash\{a\}$. When the universe $V$ is clear from the context, we let $\bar{I} \stackrel{\text { def }}{=} V \backslash I$. We often abuse notation $A+B \stackrel{\text { def }}{=} A \cup B$ for better readability.

Matroid: A tuple $\mathcal{M}=(V, \mathcal{I})$ for finite set $V$ and $\mathcal{I} \subseteq 2^{S}$ is called a matroid if (i) for every $A \in \mathcal{I}$ and $B \in \mathcal{I}$ where $|A|<|B|$, there exists an element $a \in B \backslash A$ such that $A \cup a \in \mathcal{I}$, (ii) $\emptyset \in \mathcal{I}$, and (iii) for every $A^{\prime} \subseteq A$ where $A \in \mathcal{I}$, we have $A^{\prime} \in \mathcal{I}$.

Independent Sets: We call $S \subseteq V$ independent if $S \in \mathcal{I}$ and dependent otherwise. Further, for any $S \in \mathcal{I}$ we let free $\mathcal{M}(S) \stackrel{\text { def }}{=}\{a \in \bar{S} \mid S+a \in \mathcal{I}\}$.

Matroid Polytope: The matroid polytope $\mathcal{P}_{\mathcal{M}}$ is the convex hull of the indicator vectors of the independent sets of $\mathcal{M}$.

Matroid Intersection Polytope: The matroid intersection polytope is the convex hull of the indicator vectors of the common independent sets of $\mathcal{M}_{1}, \mathcal{M}_{2}$. It is well known that this polytope is given by $\mathcal{P}_{\mathcal{M} 1} \cap \mathcal{P}_{\mathcal{M} 2}$. 
Rank: For a matroid $\mathcal{M}=(V, \mathcal{I})$ we define the rank of $\mathcal{M}$ as $\operatorname{rank}(\mathcal{M})=\max _{S \in \mathcal{I}}|S|$. Further, for any $S \subseteq V$ we define $\operatorname{rank}_{\mathcal{M}}(S) \stackrel{\text { def }}{=} \max _{T \subseteq S \mid T \in I}|T|$, i.e., the size of the largest independent set contained in $S$.

Circuits: For matroid $\mathcal{M}=(V, \mathcal{I})$ we call $S \subseteq V$ a circuit if it is a minimal dependent set, i.e., $S \notin \mathcal{I}$ but $S-a \in \mathcal{I}$ for any $a \in S$. For $S \in \mathcal{I}$ and $a \in \bar{S}$ such that $S+a \notin \mathcal{I}$ we let circuit $\mathcal{M}(S+a)$ denote a minimal dependent subset of $S+a$.

Exchangeable Pairs: For a matroid $\mathcal{M}=(V, \mathcal{I})$ and $S \in \mathcal{I}$ we call $a \in S$ and $b \notin S$ exchangeable if $S-a+b \in \mathcal{I}$. For a set $S \in \mathcal{I}$ we let exchange $\mathcal{M}_{\mathcal{M}}(S) \stackrel{\text { def }}{=}\{(a, b) \in S \times \bar{S} \mid S-a+b \in \mathcal{I}\}$ denote all exchangeable pairs in $S$.

Exchange Graph: For matroids $\mathcal{M}_{1}=\left(V, \mathcal{I}_{1}\right)$ and $\mathcal{M}_{2}=\left(V, \mathcal{I}_{2}\right)$ and $S \in \mathcal{I}_{1} \cap \mathcal{I}_{2}$ we define the exchange graph $G(S)=\left(V \cup\{s, t\}, E_{S}\right)$ as the directed graph with vertices $V \cup\{s, t\}$ where $s$ and $t$ are known as the source and sink vertices respectively. There are the following 4 types of arcs in $E_{S}:(1)(s, a)$ for all $a \in$ free $_{1}(S),(2)(a, t)$ for all $a \in$ free $_{2}(S),(3)(a, b)$ for all $a \in S$ and $(a, b) \in$ exchange $_{1}(S),(4)(b, a)$ for all $a \in S$ and $(a, b) \in$ exchange $_{2}(S)$, where for $i \in\{1,2\}$ we use free $_{i}$ and exchange ${ }_{i}$ as shorthand for free $\mathcal{M}_{i}$ and exchange $\mathcal{M}_{i}$ respectively.

Distances: For any directed graph $G=(V, E)$ and $a, b \in V$ we let $d_{G}(a, b)$ denote the shortest path distance from $a$ to $b$ using the edges of $E$. We define $d_{G}(a, a)=0$ for all $a \in V$ and if there is no $a$ to $b$ path then we let $d_{G}(a, b)=\infty$.

Distance sets: For the exchange graph $G(S)$ we denote the set of vertices at distance $i$ from source $s$ by $D_{i}$ or $L_{i}$.

Packing number: For a matroid $\mathcal{M}$ we let $\operatorname{PackNum}(\mathcal{M})$ denote the maximum number of disjoint bases in $\mathcal{M}$.

Oracles: Throughout this paper we assume that given a matroid $\mathcal{M}=(V, \mathcal{I})$ we can only access it through an oracle. We consider two such oracle models. The first, is an independence oracle, which when queried with any $S \subseteq V$, determines whether or not $S \in \mathcal{I}$ in time $\mathcal{T}_{\text {ind }}(\mathcal{M})$. The second, is a rank oracle, which when queried with an $S \subseteq V$, returns $\operatorname{rank}_{\mathcal{M}}(S)$ in time $\mathcal{T}_{\text {rank }}(\mathcal{M})$. Note that for $S \subseteq V$ we have that $S \in \mathcal{I}$ if and only if $\operatorname{rank}_{\mathcal{M}}(S)=|S|$ and consequently $\mathcal{T}_{\text {ind }}(\mathcal{M})=O\left(\mathcal{T}_{\text {rank }}(\mathcal{M})\right)$. In the typical setting where we have two matroids $\mathcal{M}_{1}$ and $\mathcal{M}_{2}$ we let $\mathcal{T}_{\text {rank }} \stackrel{\text { def }}{=} O\left(\mathcal{T}_{\text {rank }}\left(\mathcal{M}_{1}\right)+\mathcal{T}_{\text {rank }}\left(\mathcal{M}_{2}\right)\right)$ and $\mathcal{T}_{\text {ind }} \stackrel{\text { def }}{=} O\left(\mathcal{T}_{\text {ind }}\left(\mathcal{M}_{1}\right)+\mathcal{T}_{\text {ind }}\left(\mathcal{M}_{2}\right)\right)$.

\subsection{Matroid Theory}

Here we provide various standard results about the the structure of matroids and matroid intersection used throughout the paper. The first two lemmas are folklore.

Lemma 5. For matroid $\mathcal{M}=(V, \mathcal{I})$ and any $S \in \mathcal{I}$ and $a \in V$ with $S+a \notin \mathcal{I}$, we have

$$
\operatorname{circuit}_{\mathcal{M}}(S+a)=\{b \in S+a \mid S+a-b \in \mathcal{I}\} .
$$

Lemma 6 (Shortest augmenting paths). Let $s, v_{1}, \ldots v_{a}, t$ be a shortest path from $s$ to $t$ in the exchange graph $G(S)$. Then $S+v_{1}-v_{2}+\ldots-v_{a-1}+v_{a} \in \mathcal{I}_{1} \cap \mathcal{I}_{2}$, i.e., augmenting along a shortest augmenting path preserves independence. Sometimes we call this an augmentation for brevity. 
The next three lemmas will be used extensively in our algorithms. The first two show that to solve matroid intersection approximately, it suffices to stop when the length of the shortest augmenting path is $1 / \epsilon$. The last lemma imposes control over the structure of the exchange graph as we carry out augmentations.

Lemma 7 (Cunningham [6]). For any two matroids $\mathcal{M}_{1}=\left(V, \mathcal{I}_{1}\right)$ and $\mathcal{M}_{2}=\left(V, \mathcal{I}_{2}\right)$ with the largest common independent set of size $r$, given a set $S \in \mathcal{I}_{1} \cap \mathcal{I}_{2}$ of size $|S|<r$, there exists an augmenting path in $G(S)$ of size at most $1+2|S| /(r-|S|)$.

Corollary $8([6,3,21])$. For any two matroids $\mathcal{M}_{1}=\left(V, \mathcal{I}_{1}\right)$ and $\mathcal{M}_{2}=\left(V, \mathcal{I}_{2}\right)$ with the largest common independent set of size $r$, if the length of the shortest augmenting path in exchange graph $G(S)$ for some $S \in \mathcal{I}_{1} \cap \mathcal{I}_{2}$ is at least $1 / \epsilon$, then $|S| \geq(1-O(\epsilon)) \cdot r$.

Our algorithms will rely on the following monotonicity lemma of Cunningham, which says augmenting along the shortest path in an exchange graph can only increase the distance of every element from the source and sink vertices.

Lemma 9 (Monotonicity Lemma ${ }^{2}$, Cunningham [6], Price [32], Haselmayr [19]). For any two matroids $\mathcal{M}_{1}=\left(V, \mathcal{I}_{1}\right)$ and $\mathcal{M}_{2}=\left(V, \mathcal{I}_{2}\right)$ if we augment along the shortest path in $G(S)$ obtaining $G\left(S^{\prime}\right)$ for a new set $S^{\prime} \in \mathcal{I}_{1} \cap \mathcal{I}_{2}$ with $\left|S^{\prime}\right|>|S|$. Denote $d=d_{G(S)}$ and $d^{\prime}=d_{G\left(S^{\prime}\right)}$. Then for all $a \in V$,

(i) If $d(s, a)<d(s, t)$, then $d^{\prime}(s, a) \geq d(s, a)$. Similarly, if $d(a, t)<d(s, t)$, then $d^{\prime}(a, t) \geq d(a, t)$.

(ii) If $d(s, a) \geq d(s, t)$, then $d^{\prime}(s, a) \geq d(s, t)$. Similarly, if $d(a, t) \geq d(s, t)$, then $d^{\prime}(a, t) \geq d(s, t)$.

Proof. As $s$ and $t$ are symmetric, it suffices to prove the lemma for $s$. Let $p$ be the augmenting path. (i) of the lemma follows from $[19,32]$.

For (ii), suppose that there is some $a$ for which $d(s, a) \geq d(s, t)$ and $d^{\prime}(s, a)<d(s, t)$. Pick such $a$ with the minimal $d^{\prime}(s, a)$. Since $d(s, a) \geq d(s, t), a \notin p$.

Consider the vertex $b$ right before $a$ on the shortest $s-a$ path in $G\left(S^{\prime}\right)$. We have $d^{\prime}(s, b)=$ $d^{\prime}(s, a)-1<d(s, t)-2$. By the minimality of $a, d(s, b)<d(s, t)$ so (1) applies to $b$. Thus $d(s, b) \leq d^{\prime}(s, b)<d(s, t)-2$ which implies $(b, a) \notin G(S)$ (otherwise $\left.d(s, a)<d(s, t)-1\right)$. We have two cases.

As exchanging $a, b$ in $S^{\prime}$ preserves independence, and that $S$ itself is independent, $G(S)$ must contain an edge from $b$ or some internal vertex on $p$ to $a$. This is a contradiction as $(b, a) \notin G(S)$.

\section{Exchange Graph Exploration via Binary Search}

In this section we show how to use rank and independence oracles to efficiently query the exchange graph for a pair of matroids, $\mathcal{M}_{1}=\left(V, \mathcal{I}_{1}\right)$ and $\mathcal{M}_{2}=\left(V, \mathcal{I}_{2}\right)$. These graph exploration primitives form the basis of our exact and approximate matroid intersection algorithms with a rank oracle as well as our exact matroid intersection algorithm with an independence oracle.

\footnotetext{
${ }^{2}$ Throughout the paper, we will apply this lemma only on vertices $a$ which lie on a shortest $s, t$-path and therefore the conditions $d_{G(S)}(s, a)<d_{G(S)}(s, t)$ and $d_{G(S)}(a, t)<d_{G(S)}(s, t)$ hold. Furthermore, the second part shows that vertices with distance more than that cannot be part of an augmenting path of this length in the future. Cunningham's original result doesn't include this condition, and is false without it as corrected by [19, 32], which is the first part of the lemma. Our version has an extra second part which is in fact also needed for proving the correctness of Cunningham's method. We thank Troy Lee for making us aware of these conditions.
} 
In Section 3.1 we show how to efficiently find free vertices using a rank oracle and in Section 3.2 we show how to efficiently find exchangeable pairs using an independence oracle. Each routine works by a careful binary search with the appropriate oracle and serves an important role in efficiently finding edges in the exchange graph.

\subsection{Finding Free Vertices using Rank Oracle}

We show that given a matroid $\mathcal{M}=(V, \mathcal{I})$, an independent set $S \in \mathcal{I}$, and a set $B \subseteq V$, we can efficiently find a free element $e \in B$ with respect to $S$ (i.e., $S+e \in \mathcal{I}$ ) or conclude that there is none. The procedure FindFree given in Algorithm 1 finds this element by binary search with the rank oracle. There is a free element of $B$ if and only if $\operatorname{rank}_{\mathcal{M}}(B \cup S)>\operatorname{rank}(S)$ and consequently we can simply repeatedly divide $B$ in half, querying if this property still holds. Formally, we analyze the performance of this algorithm in Lemma 10.

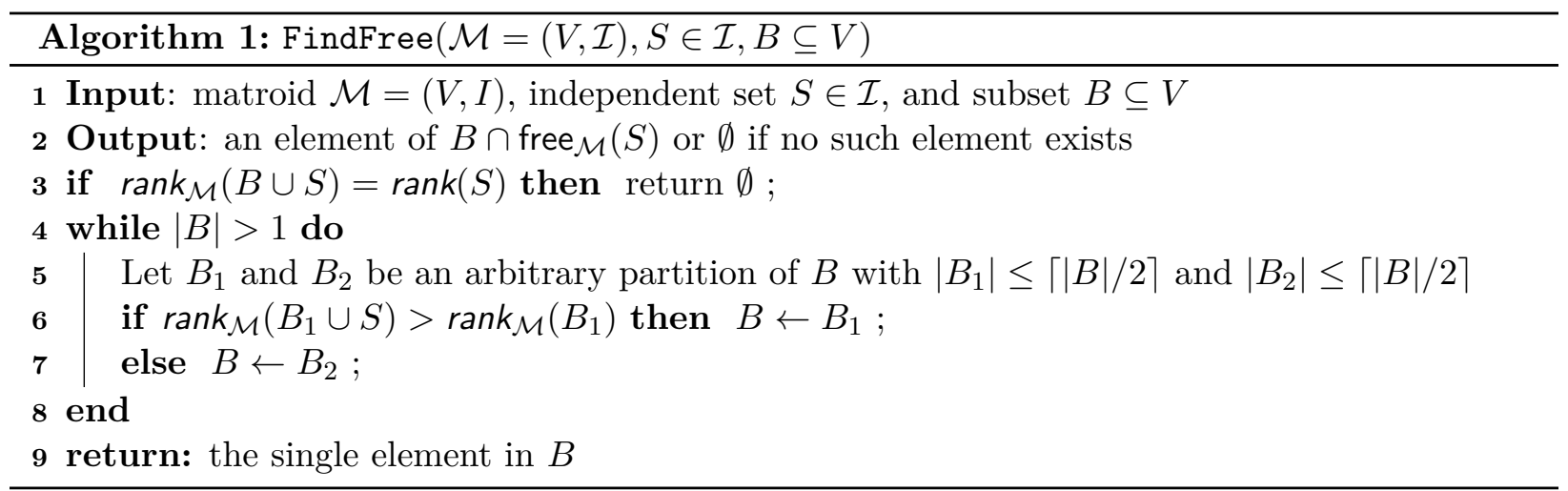

Lemma 10 (Finding Free Vertices). Given matroid $\mathcal{M}=(V, I)$, independent set $S \in \mathcal{I}$, and elements $B \subseteq V$, the procedure FindFree $(\mathcal{M}, S, B)$ (Algorithm 1) outputs in $O\left(\log (|B|) \cdot \mathcal{T}_{\text {rank }}\right)$ time either an element of $B \cap \operatorname{free}_{\mathcal{M}}(S)$, or $\emptyset$ if no such element exists.

Proof. Note that free $\mathcal{M}_{\mathcal{M}}(S) \cap B \neq \emptyset$ if and only if $\operatorname{rank}_{\mathcal{M}}(S \cup B)>\operatorname{rank}_{\mathcal{M}}(S)$. Further, if $B_{1}$ and $B_{2}$ partition $B$ and $\operatorname{rank}_{\mathcal{M}}(S \cup B)>\operatorname{rank}_{\mathcal{M}}(S)$ then either $\operatorname{rank}_{\mathcal{M}}\left(S \cup B_{1}\right)>\operatorname{rank}_{\mathcal{M}}(S)$ or $\operatorname{rank}_{\mathcal{M}}\left(S \cup B_{2}\right)>\operatorname{rank}_{\mathcal{M}}(S)$. Consequently, the output of the algorithm is correct. Since the size of $|B|$ halves in each iteration of the while loop and the partitions can be done arbitrarily, the running time is also as desired.

Given an independent set $S \in \mathcal{I}_{1} \cap \mathcal{I}_{2}$ for matroids $\mathcal{M}_{1}=\left(V, \mathcal{I}_{1}\right)$ and $\mathcal{M}_{2}=\left(V, \mathcal{I}_{2}\right)$ and an element $a \in S$, FindFree gives us an efficient way to find both incoming and outgoing arcs of $a$ in the exchange graph $G(S)$. Further, it gives an efficient way to find arcs incident to $s$ and $t$ in the exchange graph. We leverage this procedure for this purpose in Section 4.

\subsection{Finding Exchange Vertices using Independence or Rank Oracle}

We show that given a matroid $\mathcal{M}=(V, \mathcal{I})$, an independent set $S \in \mathcal{I}$, an element $b \in \bar{S}$, and $A \subseteq S$ we can efficiently find an element $a \in A$ that is exchangeable with $b$ (i.e., $S-a+b \in \mathcal{I}$ ) or conclude that there is none. The procedure FindExchange, given in Algorithm 2, finds this element by binary search using an independence oracle. We repeatedly divide the remaining elements into two halves and figure out adding which half preserves independence. Formally, we analyze the 
performance of this algorithm in Lemma 10.

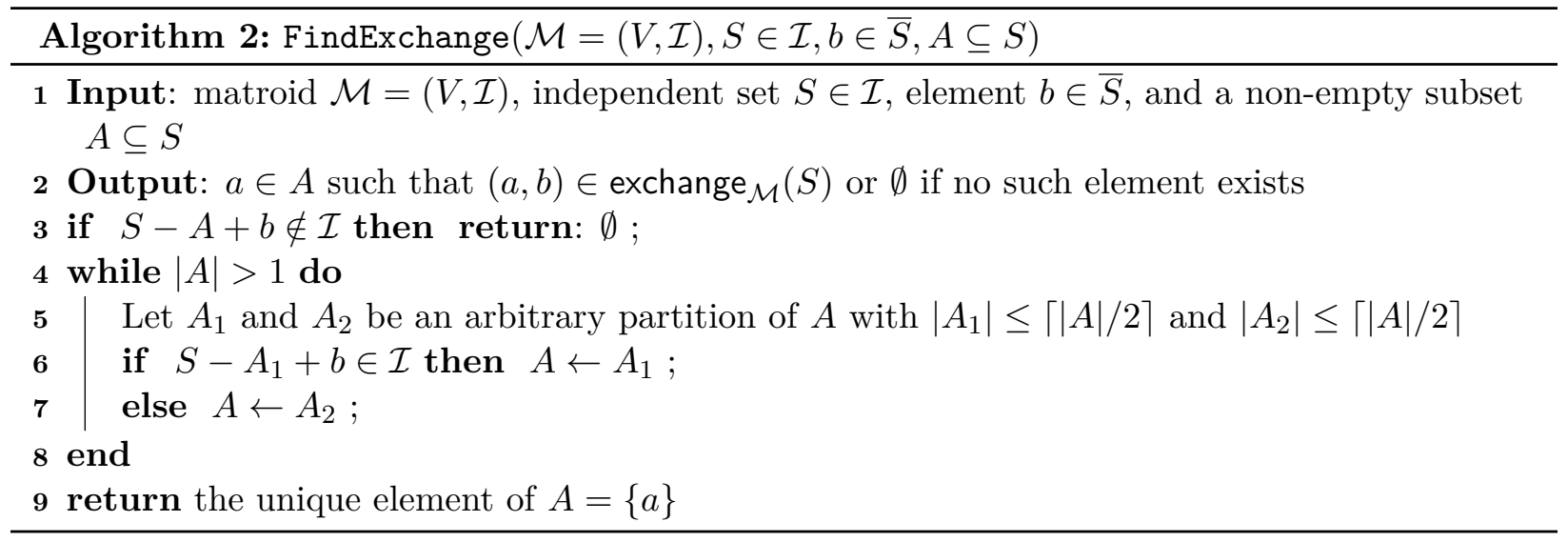

Lemma 11 (Finding Exchange Vertices). Given a matroid $\mathcal{M}=(V, \mathcal{I})$, independent set $S \in \mathcal{I}$, element $b \in \bar{S}$, and a non-empty subset $A \subseteq S$, the procedure FindExchange $(\mathcal{M}, S, b, A)$ (Algorithm 2) outputs in $O\left(\log (|A|) \cdot \mathcal{T}_{\text {ind }}\right)=O\left(\log r \cdot \mathcal{T}_{\text {ind }}\right)$ time either an element a $\in$ A such that $(a, b) \in$ exchange $_{\mathcal{M}}(S)$, or $\emptyset$ if no such element exists

Proof. If $S-A+b \notin \mathcal{I}$ then of course no such $a$ exists. Assume $S-A+b \in \mathcal{I}$.

By considering $S \in \mathcal{I}$, we can add $|A|-1$ elements of $A$ to $S-A+b$ while preserving independence. In particular, for any partition of $A$ into nonempty $A_{1}, A_{2}$, we must have $S-A_{1}+b \in \mathcal{I}$ or $S-A_{2}+b \in \mathcal{I}$. This proves the correctness of FindExchange $(\mathcal{M}, S, b, A)$ which just repeatedly performs this.

Since we halve the size of $A$ in each iteration, there can be at most $O(\log |A|)$ iterations and the runtime follows.

Given an independent set $S \in \mathcal{I}_{1} \cap \mathcal{I}_{2}$ for matroids $\mathcal{M}_{1}=\left(V, \mathcal{I}_{1}\right)$ and $\mathcal{M}_{2}=\left(V, \mathcal{I}_{2}\right)$ and an element $a \in \bar{S}$, FindExchange gives us an efficient way to find both incoming and outgoing arcs of $a$ in the exchange graph. We will leverage this procedure for this purpose in Section 5 and Section 6, where we will exploit that this procedure only requires an independence oracle.

In fact, the same procedure works for the rank oracle as well since it easily implements the independence oracle:

Lemma 12 (Finding Exchange Vertices). Given matroid $\mathcal{M}=(V, \mathcal{I})$, independent set $S \in \mathcal{I}$, element $b \in \bar{S}$, and non-empty subset $A \subseteq S$, the procedure FindExchange $(\mathcal{M}, S, b, A)$ (Algorithm 2) outputs in $O\left(\log (|A|) \cdot \mathcal{T}_{\text {rank }}\right)=O\left(\log r \cdot \mathcal{T}_{\text {ind }}\right)$ time either $a \in A$ such that $(a, b) \in$ exchange $_{\mathcal{M}}(S)$, or $\emptyset$ if no such element exists.

Proof. A set $J$ is independent iff $\operatorname{rank}(J)=|J|$, so each independence oracle call can be implemented by exactly one rank oracle call. Our result then follows directly from Lemma 11.

\section{Exact and Approximation Algorithms using Rank Oracle}

In this section we consider the matroid intersection problem in the rank oracle model. We assume throughout this section that $\mathcal{M}_{1}=\left(V, \mathcal{I}_{1}\right)$ and $\mathcal{M}_{2}=\left(V, \mathcal{I}_{2}\right)$ with $n \stackrel{\text { def }}{=}|V|$ are given by rank oracles and our goal is to provide both faster approximate and exact algorithms for finding a maximum cardinality set in $\mathcal{I}_{1} \cap \mathcal{I}_{2}$. We split the derivation of these algorithms into three parts.

In Section 4.1 we provide basic primitives for exploring the exchange graph with a rank oracle. Then, in Section 4.2 we provide the main subroutine for our intersection algorithm which efficiently 
computes a type of blocking flow in the exchange graph. Finally, in Section 4.3 we show how to use this blocking flow subroutine to obtain our desired algorithms for solving matroid intersection with a rank oracle.

\subsection{Exploring the Exchange Graph with a Rank Oracle}

Here we show how to use the algorithms FindFree (Algorithm 1) and FindExchange (Algorithm 2) of Section 3 to efficiently find edges and distances in the exchange graph. First, we provide the algorithm OutArc (Algorithm 3) which, given any vertex $a$ and set $B$ of the exchange graph, quickly finds an arc from $a$ to $B$ (if it exists). This algorithm simply proceeds case by case finding the possible arcs. If $a=s$ then the arcs can be found simply by looking for free vertices in $B$. If $a \in S$ then the arcs can be found simply by looking for vertices in $B \cap \bar{S}$ that are free with respect to $S-a$. Similarly, if $a \notin S$ and $t \in B$ then edges to $t$ can be find just by checking if $S+a$ is independent. Finally, we can use FindExchange to find arcs from $a \in \bar{S}$ to $b \in S$.

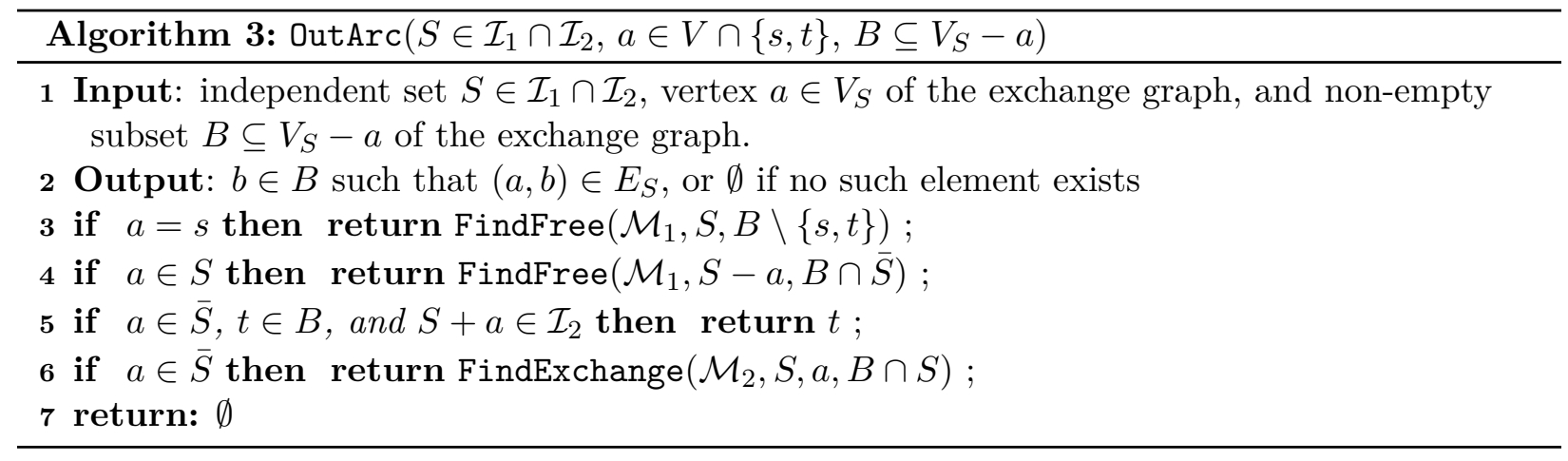

Lemma 13 (Finding Out Arcs Vertices). Given independent set $S \in \mathcal{I}_{1} \cap \mathcal{I}_{2}$, a vertex a $\in V_{S}$ of the exchange graph, and non-empty subset B, the algorithm OutArc(S,a,B) (Algorithm 3) outputs in $O\left(\log (|B|) \cdot \mathcal{T}_{\text {rank }}\right)$ time either $b \in B$ such that $(a, b) \in E_{S}$, or $\emptyset$ if no such element exists.

Proof. The correctness and runtime follow almost immediately from Lemma 10 which provides guarantees on FindFree, and Lemma 12 which provides guarantees on FindExchange. To see the correctness, simply consider the definition of the exchange graph; this algorithm directly checks for the existence of each type of edge one at a time.

Next, leveraging OutArc (Algorithm 3) and its analysis in Lemma 13 we show that we can efficiently compute all distances from $s$ in the exchange graph. The algorithm GetDistancesRank (Algorithm 4) given below achieves this goal by essentially performing breadth first search (BFS) 
in the exchange graph. For the sake of completeness, we include the details below.

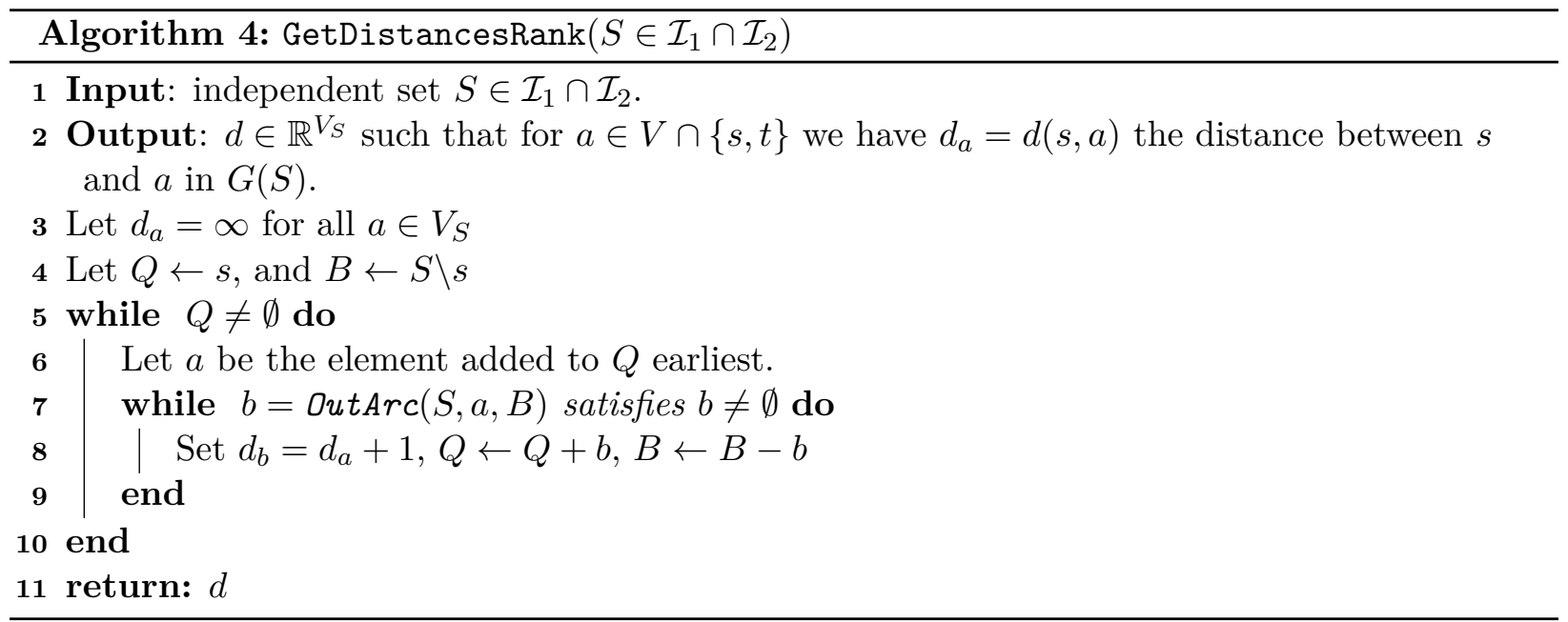

Lemma 14 (Finding Distances). Given independent set $S \in \mathcal{I}_{1} \cap \mathcal{I}_{2}$ the GetDistancesRank $(S)$ (Algorithm 3) outputs in $O\left(n \log n \cdot \mathcal{T}_{\text {rank }}\right)$ time $d \in \mathbb{R}^{V_{S}}$ such that for $a \in V_{S}$ we have $d_{a}=d(s, a)$ is the distance between $s$ and $a$ in the exchange graph $G(S)$.

Proof. The procedure GetDistancesRank simply computes distances from $s$ using breadth first search. Note that each vertex is added to $Q$ at most once and removed from $B$ at most once. Consequently, the cost of the procedure is simply $O(n)$ plus the cost of $O(n)$ calls to OutArc. So the correctness and runtime of the procedure follow from the analysis of OutArc given by Lemma 13.

\subsection{Blocking Flow with Rank Oracle}

In this section we provide our main DFS-like subroutine for improving an independent set $S \in$ $\mathcal{I}_{1} \cap \mathcal{I}_{2}$. This algorithm, BlockFlow (Algorithm 5), efficiently performs an analog of blocking flow for the exchange graph. This is essentially a fast rank-oracle implementation of each phase in Cunningham's algorithm [6].

Given a set $S \in \mathcal{I}_{1} \cap \mathcal{I}_{2}$ it first computes the distance from $s$ to every vertex in the exchange graph using GetDistancesRank. Using these distances, the algorithm subdivides $V$ into sets $L_{i}$ such that $L_{i}$ has all vertices at distance $i$ from $s$ in the exchange graph of $S$. The algorithm then uses OutArc to look for a $s, a_{1}, a_{2}, \ldots, a_{d_{t}-1}, t$ path in $G(S)$ where each $a_{i} \in L_{i}$. If found, the algorithm augments along such a path removing those vertices and updating $S$ and looking for a new path. However, whenever the algorithm concludes that some vertex in a $L_{i}$ is not on such a path (i.e., a dead end), it removes it from the graph. By carefully searching for these paths we show that ultimately this algorithm is asymptotically no more expensive then GetDistancesRank itself and will always find a larger set in $\mathcal{I}_{1} \cap \mathcal{I}_{2}$ (if it exists) where the distance from $s$ to $t$ in the 
exchange graph has increased.

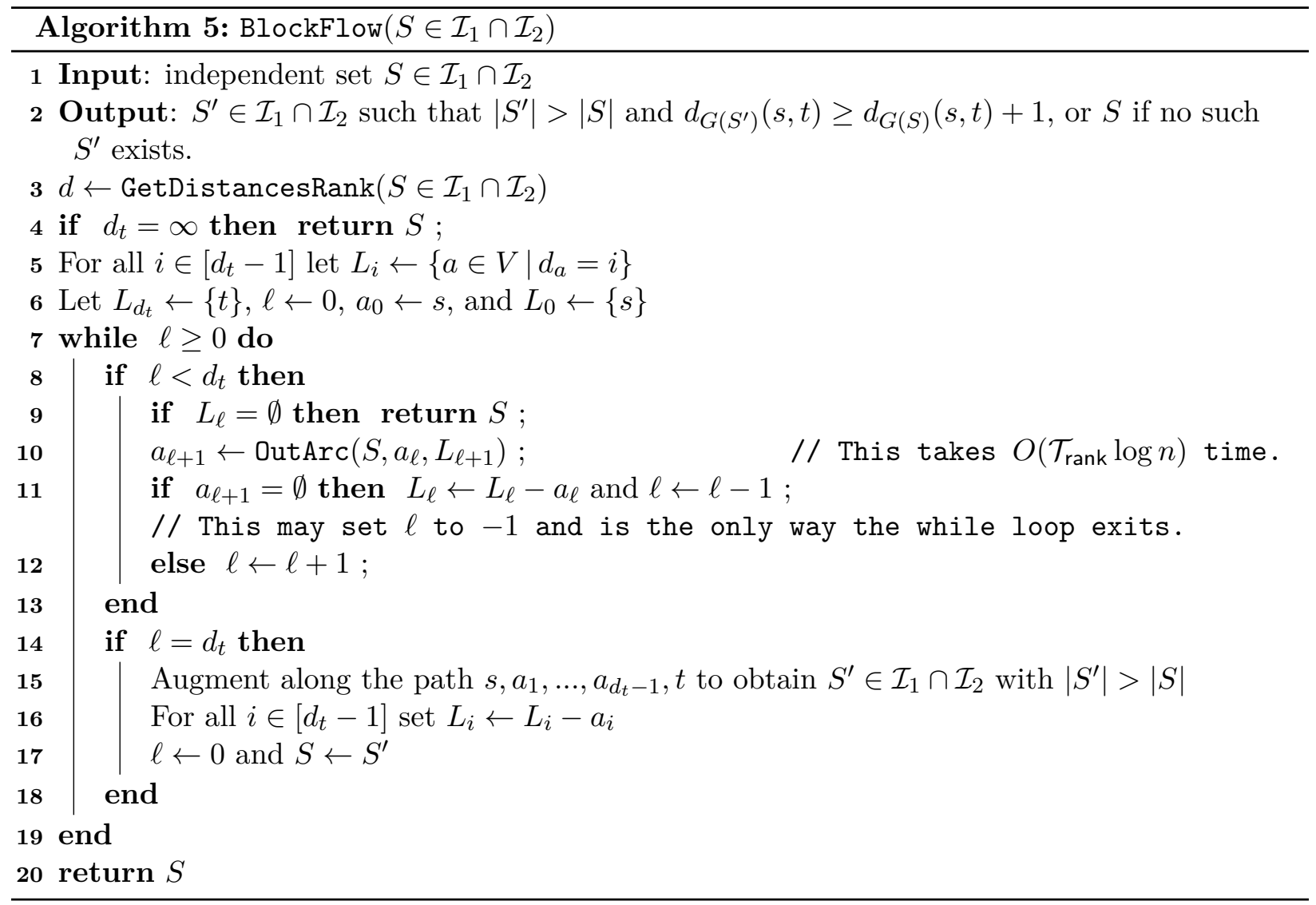

Lemma 15 (Blocking Flow). Given independent set $S \in \mathcal{I}_{1} \cap \mathcal{I}_{2}$ the algorithm BlockFlow (Algorithm 5) outputs in $O\left(n \log n \cdot \mathcal{T}_{\text {rank }}\right)$ time $S^{\prime} \in \mathcal{I}_{1} \cap \mathcal{I}_{2}$ such that $\left|S^{\prime}\right|>|S|$ and $d_{G\left(S^{\prime}\right)}(s, t) \geq$ $d_{G(S)}(s, t)+1$, or $S$ if no such $S^{\prime}$ exists (i.e., $S$ is the maximum cardinality set in $\left.\mathcal{I}_{1} \cap \mathcal{I}_{2}\right)$.

Proof. We first analyze the running time. By Lemma 14, in $O\left(n \cdot \mathcal{T}_{\text {rank }} \log n\right)$ the algorithm GetDistancesRank will ensure that $d_{a}=d_{G(S)}(s, a)$ for all $a \in V$. Once these distances are computed the algorithm spends $O(n)$ time to subdivide $V$ into the $L_{i}$.

Further, by Lemma 13 each invocation of OutArc takes $O\left(\mathcal{T}_{\text {rank }} \log n\right)$ time and finds an edge from $a_{\ell}$ to $L_{\ell+1}$ if one exists. Now, in every iteration of the while loop $\ell$ is either increased or decreased. Every time it is decreased, either it is by a value of 1 , in which case $a_{\ell}$ is removed from $L_{\ell}$ and there is no path of the form $s, a_{1}, \ldots, a_{d_{t}-1}, t$ where each $a_{i} \in L_{i}$ and $a_{\ell}$ is included, or a path is found and all the vertices on the path are removed. Consequently, there are only $O(n)$ iterations of the while loop and the total cost of the algorithm is as desired.

Now we show $S^{\prime} \in \mathcal{I}_{1} \cap \mathcal{I}_{2}$. The reasoning in the preceding paragraph shows that this algorithm simply finds a $s$ to $t$ path through the $L_{i}$ if there is one, removing those vertices, and repeating until there are no more paths. Such augmenting paths are of length $d_{t}$ by design and hence they are in fact shortest augmenting paths. This implies $S^{\prime} \in \mathcal{I}_{1} \cap \mathcal{I}_{2}$ as augmenting along a shortest augmenting path preserves independence (Lemma 6). Further there must be at least one augmentation since GetDistancesRank computed $d_{t}$. So $\left|S^{\prime}\right|>|S|$.

It remains to show $d_{G\left(S^{\prime}\right)}(s, t) \geq d_{G(S)}(s, t)+1$. This is similar to the argument in Cunningham [6]. We argue that there is no more augmenting path of length $d_{t}$ and consequently the distance from $s$ to $t$ must increase. To this end, we must prove that any elements removed from $L_{i}$ can no longer be on any augmenting path of length $d_{t}$. 
By Lemma 9, distance of every vertex from $s$ and to $t$ increases monotonically after each such augmentation. There are two ways a vertex is removed. In the former case $a_{\ell}$ has no outgoing arc into $L_{\ell+1}$ and hence is a dead end. In the latter case they are on an augmenting path of length $d_{t}$.

For the elements on such a path, since they enter or exit $S$ after an augmentation, their distances from $s$ and to $t$ must strictly increase. Consequently they cannot be on such a path anymore. Further, since monotonicity implies that to stay on a $s$ to $t$ path of distance $d_{G(S)}(s, t)$ for the original $G$ their distance from $s$ cannot increase this implies that every vertex removed is not on a $s$ to $t$ path of length $d_{t}$.

\subsection{Matroid Intersection with a Rank Oracle}

In this section we show how to use BlockFlow (Algorithm 5) from the previous section to obtain our desired rank oracle based algorithms for solving matroid intersection. All our algorithms simply apply BlockFlow repeatedly to find the desired set. First, we provide our result about exactly solving matroid intersection with a rank oracle and then we provide our approximate result.

Both of our algorithms follow the structure of Cunningham's [6]. The key difference is our fast subroutine BlockFlow for handling each phase.

Theorem 16 (Exact Rank Oracle Algorithm). Given matroids $\mathcal{M}_{1}=\left(V, \mathcal{I}_{1}\right)$ and $\mathcal{M}_{2}=\left(V, \mathcal{I}_{2}\right)$ there is an algorithm which finds the largest common independent set of two matroids in $O(n \sqrt{r} \log n$. $\left.\mathcal{T}_{\text {rank }}\right)$ time where $r$ is the size of the largest common independent set.

Proof. Our algorithm simply starts with $S_{0}=\emptyset$ and iterates $S_{i+1}:=\operatorname{BlockFlow}\left(S_{i}\right)$ until $S_{k+1}=S_{k}$ for some $k$ at which point the algorithm outputs $k$. By Lemma 15 we have that $S_{i} \in \mathcal{I}_{1} \cap \mathcal{I}_{2}$ for all $\mathcal{I}$ and that $S_{k}$ is the desired largest common independent set. Further, Lemma 15 implies that the running time of this algorithm is $O\left(n k \mathcal{T}_{\text {rank }} \log n\right)$. Consequently, it only remains to bound $k$.

Now, Lemma 15 also implies that for all $i<k$ we have $d_{G\left(S_{i+1}\right)}(s, t) \geq d_{G\left(S_{i}\right)}(s, t)+1$. So $d_{G\left(S_{i}\right)} \geq i$ for all $i \in[k]$. Further, Corollary 8 implies that $\left|S_{i}\right| \geq(1-O(1 / i)) r$. Consequently, $i=\Omega(\sqrt{r})$ implies that $\left|S_{i}\right| \geq r-O(\sqrt{r})$. Since every iteration of blocking flow increases the size of $\left|S_{i}\right|$ this implies that increasing $i$ by another $O(\sqrt{r})$ is enough to get a set in $\mathcal{I}_{1} \cap \mathcal{I}_{2}$ of size $r$. Consequently, $k=O(\sqrt{r})+O(\sqrt{r})=O(\sqrt{r})$.

Theorem 17 (Approximate Rank Oracle Algorithm). Given matroids $\mathcal{M}_{1}=\left(V, \mathcal{I}_{1}\right)$ and $\mathcal{M}_{2}=$ $\left(V, \mathcal{I}_{2}\right)$ there is an algorithm which finds a $(1-\epsilon)$ approximation to the largest common independent set of the two matroids in time $O\left(n \epsilon^{-1} \log n \cdot \mathcal{T}_{\text {rank }}\right)$.

Proof. Similar to proof of Theorem 16 our algorithm simply starts with $S_{0}=\emptyset$ and iterates $S_{i+1}:=$ BlockFlow $\left(S_{i}\right)$. However, here instead of repeating until $S_{k+1}=S_{k}$ we simply output $S_{k}$ for $k=\Theta\left(\epsilon^{-1}\right)$. Again, by Lemma 15 we immediately have that the runtime is as desired and we have that $S_{i} \in \mathcal{I}_{1} \cap \mathcal{I}_{2}$ and $d_{G\left(S_{i+1}\right)}(s, t) \geq d_{G\left(S_{i}\right)}(s, t)+1$ for all $i$. Since this implies $d_{G\left(S_{k}\right)}=\Omega\left(\epsilon^{-1}\right)$, we have by Corollary 8 that $\left|S_{k}\right| \geq(1-\epsilon) r$ where $r$ is the size of the largest common independent set of two matroids and we have the desired result.

In fact, both of our exact and approximate results hold even if we have access to a rank oracle for one matroid and access to only an independence oracle for the second. This is because FindExchange requires only an independence oracle. We omit the detials. 


\section{Exact Algorithm using Independence Oracle}

In this section we show how to solve matroid intersection exactly using independence oracles. We assume throughout this section that we are given matroids $\mathcal{M}_{1}=\left(V, \mathcal{I}_{1}\right)$ and $\mathcal{M}_{2}=\left(V, \mathcal{I}_{2}\right)$ with $n \stackrel{\text { def }}{=}|V|$ and they can only be accessed via an independence oracle. The goal is to compute the largest common independent set and the following is our main result of this section.

Theorem 18. We can find the largest common independent set of two matroids using $O(n r \log r$.

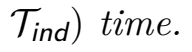

To recall, the main challenge with independence oracles is that we do not have $O\left(\mathcal{T}_{\text {ind }} \log n\right)$ oracles to implement the FindFree subroutine. Nevertheless, we can obtain a speed-up using binary search ideas.

The run time of this algorithm can be broken into two components. First, we show in Section 5.1 that given any common independent set $S$, we can find the distance of every element from $s$ in the exchange graph $G(S)$ in $O\left(n \log r \cdot \mathcal{T}_{\text {ind }}\right)$ time plus an "amortized" cost. This amortized cost will turn out to be $O\left(\log r \cdot \mathcal{T}_{\text {ind }}\right)$ per element whenever its distance increases from the sources. In our final AugmentingPaths algorithm in Section 5.3 we will exploit the fact that this increase in distance can happen at most $O(r)$ times for an element, so the overall amortized cost over all elements is $O\left(n r \log r \cdot \mathcal{T}_{\text {ind }}\right)$.

Second, given the distances in the exchange graph $G(S)$, in Section 5.2 we spend $O\left(n \log r \cdot \mathcal{T}_{\text {ind }}\right)$ time to find one augmenting path. Since there can be at most $r$ augmentations during the entire execution of the algorithm, this second component will also be at most $O\left(n r \log r \cdot \mathcal{T}_{\text {ind }}\right)$.

\subsection{Finding the Distances}

We show how to compute all distances from $s$ in the exchange graph. The following algorithm GetDistancesIndep (Algorithm 6) achieves this goal simply by proceeding case by case. Starting with the source $s$, it finds the distances by running a variant of BFS. Going from an odd layer $\ell=2 k+1$ to layer $\ell+1$ is easy as we can just use the FindExchange algorithm. To go from even layer $\ell=2 k$ to $\ell+1$, the idea is that we know up till $D_{\ell}$ where $D_{\ell}=\left\{a \in V \mid d_{a}=\ell\right\}$, and that we maintain some candidates $L_{\ell+1}$ for $D_{\ell+1}$ (here $L_{\ell+1}$ are elements we know are at distance at least $\ell+1$ from the source). Consider any such candidate element $v \in L_{\ell+1}$. By one call to FindExchange we can determine if $v$ has a neighbor in $D_{\ell}$. If yes, then we know $v \in D_{\ell+1}$, otherwise 
it is at distance at least $\ell+3$, so we move it to $L_{\ell+3}$.

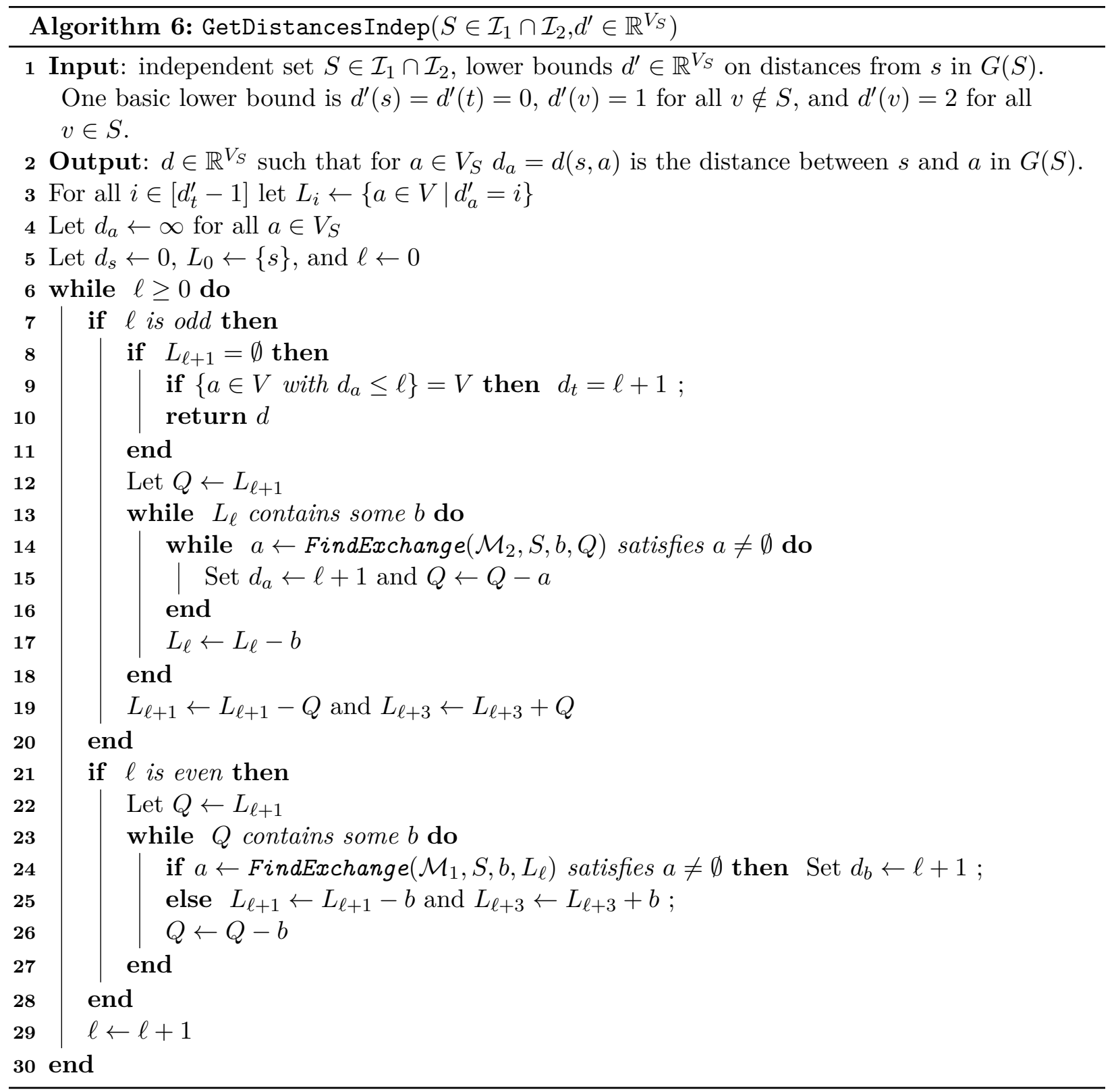

Lemma 19 (Finding Distances). Given independent set $S \in \mathcal{I}_{1} \cap \mathcal{I}_{2}$ and distance lower bounds $d^{\prime} \in$ $\mathbb{R}^{V_{S}}$ for $G(S)$, i.e., for $a \in V_{S}$ we know $d^{\prime}(s, a) \leq d_{G(S)}(s, a)$. The algorithm GetDistancesIndep $\left(S, d^{\prime}\right)$ (Algorithm 6) outputs $d \in \mathbb{R}^{V_{S}}$ in $O\left(\sum_{a \in V}\left(1+d_{a}-d_{a}^{\prime}\right) \log r \cdot \mathcal{T}_{\text {ind }}\right)$ time such that for $a \in V_{S}$ we have $d_{a}=d_{G(S)}(s, a)$.

Proof. To prove correctness of GetDistancesIndep, we prove the following invariant at the beginning of any iteration of the outer while loop.

1. $D_{i} \subseteq \bigcup_{j \leq i} L_{j}$ for $i>\ell$ and $L_{\ell}=D_{\ell}$.

2. $d$ gives correct distances of elements at distance at most $\ell$ from the source $s$. 
Clearly the invariant implies correctness of the algorithm when we take $\ell=d_{G(S)}(s, t)$. The invariant is true for $\ell=0$ from the definition of $d^{\prime}$ and as we set $d_{s}=0$. To prove the invariant for $\ell+1$, we separately consider $\ell$ being even or odd.

When $\ell$ is odd the algorithm essentially performs BFS. It considers vertices in $L_{\ell}$ one-by-one and check which of the vertices in $L_{\ell+1}$ are reachable. All vertices that we can reach in $L_{\ell+1}$ are definitely at distance $\ell+1$ and the remaining vertices are at distance at least $\ell+3$.

When $\ell$ is even the algorithm considers every vertex in $L_{\ell+1}$ one-by-one and check if it is reachable from $L_{\ell}$. All vertices that we can reach from $L_{\ell}$ are definitely at distance $\ell+1$, and the remaining vertices are at distance at least $\ell+3$ so we update $L_{\ell+3}$ accordingly.

To analyze the running time of the algorithm, consider any element $a$. Suppose it was initially in $L_{i}$ according to $d^{\prime}$ and is finally at distance $j$ according to $d$. Observe that we change the layers of this element at most $\lceil(j-i) / 2\rceil$ times. In each of these changes, we spend at most one call to FindExchange. By Lemma 11 each such call takes $O\left(\mathcal{T}_{\text {ind }} \log r\right)$ time, which means the overall time taken by the algorithm is $O\left(\sum_{a \in V}\left(1+d_{a}-d_{a}^{\prime}\right) \log r \cdot \mathcal{T}_{\text {ind }}\right)$.

\subsection{Finding One Augmenting Path}

Next, given distances $d$ from $s$ to every vertex in the exchange graph using GetDistancesRank, we show how to find a single augmenting path in $O\left(n \mathcal{T}_{\text {ind }}\right)$ time. Let $D_{i}=\left\{a \in V \mid d_{a}=i\right\}$.

The idea is to start with $\ell=d_{t}$ at the sink $t$ and perform a depth-first search. To go from $\ell$ to $\ell-1$, i.e, given a vertex $v \in D_{\ell}$, to find an adjacent vertex in $D_{\ell-1}$, we try every possible vertex in $D_{\ell-1}$ and check if it has an edge to $v$. Notice that we are guaranteed to find some vertex $u \in D_{\ell-1}$ with edge $(u, v)$ in $G(S)$ because $v \in D_{\ell}$. By continuing this procedure, we are guaranteed to find an augmenting path of length $d_{t}$. Since we reach or check each vertex at most once, the overall running time of finding this augmenting path will be $O(n)$.

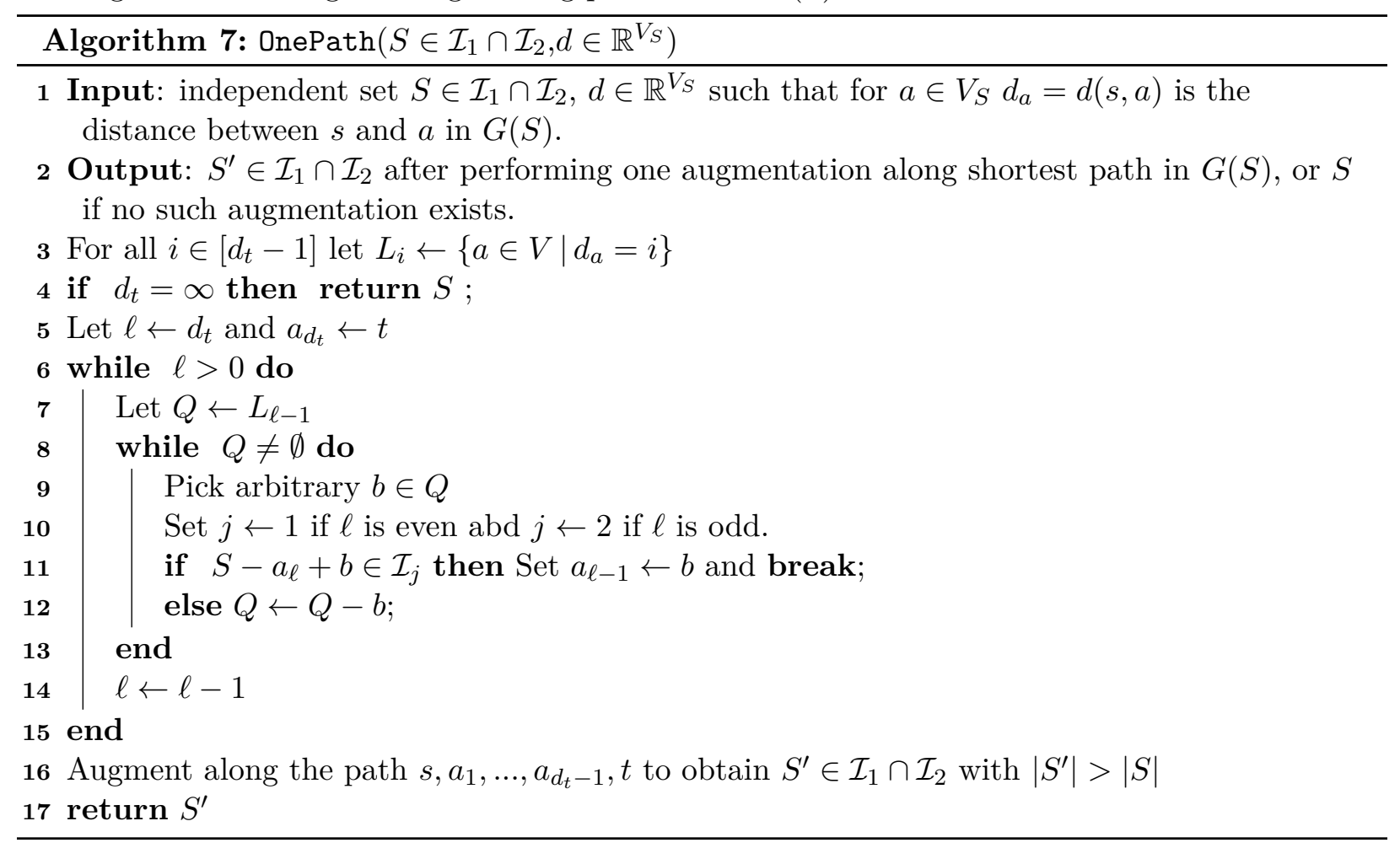


Lemma 20 (One Augmentation). Given independent set $S \in \mathcal{I}_{1} \cap \mathcal{I}_{2}$ and distance $d \in \mathbb{R}^{V_{S}}$ such that for $a \in V_{S}, d_{a}=d(s, a)$ is the distance between $s$ and $a$ in the exchange graph $G(S)$, the algorithm OnePath $(S, d)$ (Algorithm 7) outputs $S^{\prime} \in \mathcal{I}_{1} \cap \mathcal{I}_{2}$ with $\left|S^{\prime}\right|>|S|$ in $O\left(n \cdot \mathcal{T}_{\text {ind }}\right.$ ) time by performing an augmentation along a shortest path in $G(S)$, or $S$ if there is no such $S^{\prime}$.

Proof. Starting with the sink vertex $t$, the algorithm iteratively finds an element $a_{\ell-1}$ at distance $\ell-1$ from $s$ such that there is an edge $\left(a_{\ell-1}, a_{\ell}\right)$. It tries every vertex $b \in L_{\ell-1}$ and check if there is an edge $(b, a)$. We are guaranteed to find some $b$ because $a_{\ell}$ is at distance $\ell$ from $s$. The time taken by this step is $O\left(\left|L_{\ell-1}\right| \cdot \mathcal{T}_{\text {ind }}\right)$. Since $\sum_{\ell}\left|L_{\ell-1}\right| \leq n$, the overall time taken by the algorithm is $O\left(n \cdot \mathcal{T}_{\text {ind }}\right)$.

\subsection{The Augmenting Paths Algorithm}

Now we use the subroutines from the last section to find the largest common independent set in any two given matroids and prove Theorem 18. The AugmentingPaths algorithms alternates between GetDistancesIndep and OnePath algorithms to perform augmentations.

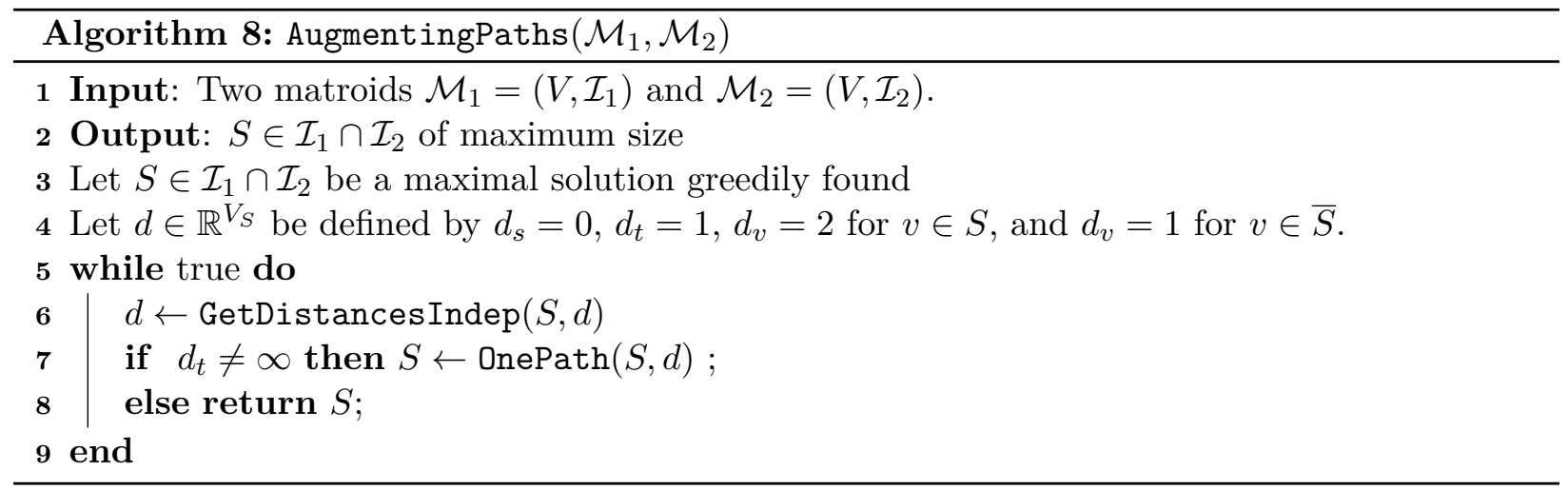

Proof of Theorem 18. Starting with a feasible solution $S$, since the AugmentingPaths algorithm finds all shortest augmenting paths one-by-one, it clearly returns the optimal solution.

Next we bound the running time. The maximal common independent set $S \in \mathcal{I}_{1} \cap \mathcal{I}_{2}$ can be greedily computed by adding an element $e$ to $S$ if $S+e \in \mathcal{I}_{1} \cap \mathcal{I}_{2}$. This only takes $O\left(n \cdot \mathcal{T}_{\text {ind }}\right)$ time. To bound the overall time taken by all calls to GetDistancesIndep we use Lemma 19. Notice that an element $a$ 's distance $d_{a}$ only increases during the execution of the algorithm. Since its final distance is at most $r$, we get the overall time is $O\left(n r \log r \cdot \mathcal{T}_{\text {ind }}\right)$. Finally, each call to OnePath takes $O\left(n \mathcal{T}_{\text {ind }}\right)$ time by Lemma 20 . Since the total number of augmentations performed using OnePath is $O(r)$, these calls take $O\left(n r \cdot \mathcal{T}_{\text {ind }}\right)$ time.

Remark. In fact we can achieve a slightly better runtime of $O\left(\left(n r+r^{2} \log r\right) \cdot \mathcal{T}_{\text {ind }}\right)$ time. When computing the distances from an even layer $L_{2 k}$ to an odd layer $L_{2 k+1}$, for each $b \in L_{2 k+1}$ we check if $b$ has an incoming edge from $L_{2 k}$ using FindExchange which takes $O\left(\log r \cdot \mathcal{T}_{\text {ind }}\right)$ time. Instead of explicitly finding such an edge, we can simply check if $S-L_{2 k}+b$ is independent and determine if $b$ has distance $2 k+1$. This requires only one independence call. We presented the slightly slower implementation because it is easier to understand.

Remark. Nguyễn's recent concurrent and independent note [11] exploits a similar binary search idea and obtains an algorithm with $O\left(n r \log ^{2} r\right)$ independence queries. The reason for the extra $\log r$ factor seems to arise because he does not amortize the cost per vertex as we do in Lemma 19. Indeed, if we do not have the $d_{a}^{\prime}$ term, then every $a \in V$ would pay $O\left(d_{a} \log r \cdot \mathcal{T}_{\text {ind }}\right)$ and it is known (can be derived from Lemma 7) that $\sum_{a} d_{a}=O(r \log r)$. This would give Nguyê̂n's factor [11]. 


\section{Approximation Algorithm using Independence Oracle}

In this section we consider the problem of obtaining a $(1-\epsilon)$-approximate common independent set in two matroids $\mathcal{M}_{1}=\left(V, \mathcal{I}_{1}\right)$ and $\mathcal{M}_{2}=\left(V, \mathcal{I}_{2}\right)$ using independence oracles. Our main result is the following theorem.

Theorem 21. There is an $O\left(\frac{n^{1.5} \sqrt{\log r}}{\epsilon^{1.5}} \cdot \mathcal{T}_{\text {ind }}\right)$-time algorithm to obtain a $(1-\epsilon)$-approximation to the matroid intersection problem.

Overview. Our algorithm uses Cunningham's idea of performing augmentations in phases. Let $S$ denote the solution of the algorithm at the start of a phase. Denote the exchange graph of $S$ by $G(S)$. Let the length of the shortest path in $G(S)$ be $2(\ell+1)$. Let $D_{k}$ be the set of elements at distance $k \leq 2(\ell+1)$ from the source $s$. Note by Lemma 19, we can compute these sets in $O\left(n \log r \cdot \mathcal{T}_{\text {ind }}\right)$ time. The rough idea of Cunningham's algorithm in a phase is to perform a maximal collection of augmentations as long as the shortest path length remains the same. Once such a maximal collection is augmented, the distance from source to sink increase by at least 2 and we move on to the next phase. Note that, by Corollary 8 , one obtains a $(1-\epsilon)$-approximate solution after $O(1 / \epsilon)$ phases. Thus the total running time of the algorithm is $O(1 / \epsilon)$ times the time (number of independence oracles) needed to process each phase. The rest of this section therefore focuses on how to process a phase fast.

In each phase, Cunningham's algorithm takes one augmenting path at a time. This becomes necessary for any "augmenting paths" style algorithm because each augmentation might add or delete several edges in the exchange graph. For example, taking one augmenting path may destroy another disjoint augmenting $\mathrm{path}^{3}$. Unfortunately, this means we cannot beat $O(n r)$ since we don't know how to do an augmentation in better than $O(n)$ time. To overcome this barrier, our main idea is to simultaneously find multiple augmenting paths. Of course this needs care as one augmenting path might destroy another path. Our crucial observation is that the union of a sequence of consecutive shortest augmenting paths can be interpreted as "augmentation sets". These augmentation sets satisfy many of the properties of an augmenting path. Although finding a maximal collection of augmenting sets still takes quadratic time, to obtain a sub-quadratic algorithm we halt this Augmenting Sets algorithm early in Section 6.4 and combine it with our Augmenting Path algorithm from Section 5.

Before describing our Augmenting Sets algorithm in Section 6.3, we start by proving some basic facts about matroids and then studying the properties of augmenting sets.

\subsection{Facts about Matroids}

Fact 22. Let $S$ be a common independent set in two matroids $\mathcal{M}_{1}=\left(V, \mathcal{I}_{1}\right)$ and $\mathcal{M}_{2}=\left(V, \mathcal{I}_{2}\right)$. Let $G(S)$ be the exchange graph w.r.t $S$. If there exists a set $A \subseteq S$ and $b \notin S$ such that there is no edge of the form $(a, b) \in E(G(S))$ for any $a \in A$, then $S-A+b \notin \mathcal{I}_{1}$. Similarly, if there is no edge of the form $(b, a) \in E(G(S))$ for any $a \in A$, then $S-A+b \notin \mathcal{I}_{2}$.

Proof. Direct from the defintion of matroids.

Fact 23. Let $\mathcal{M}=(V, \mathcal{I})$ be any matroid and let $S \in \mathcal{I}$ be an independent set. Let $X \subseteq S$ and $Y, Z \subseteq \bar{S}$ with $Y \cap Z=\emptyset$ satisfy the following properties:

\footnotetext{
${ }^{3}$ As a concrete example, consider the maximum bipartite matching problem (which is an intersection of two partition matroids) on the graph being a path $\left\{e_{1}, e_{2}, \ldots, e_{6}\right\}$ of length 6 . Suppose the current matching is $\left\{e_{2}, e_{5}\right\}$. The corresponding exchange graph has two disjoint augmenting paths $\left\{e_{1}, e_{2}, e_{3}\right\}$ and $\left\{e_{4}, e_{5}, e_{6}\right\}$. However, if we take both the augmenting paths simultaneously then the obtained set $\left\{e_{1}, e_{3}, e_{4}, e_{6}\right\}$ is not a valid matching.
} 
(a) $S+Z \in \mathcal{I}$

(b) $S-X+Y \in \mathcal{I}$

(c) $Y \in \operatorname{span}(S)$, that is, $\operatorname{rank}(S+Y)=\operatorname{rank}(S)=|S|$.

Then, $S+Z-X+Y \in \mathcal{I}$.

Proof. By submodularity of rank,

$$
\operatorname{rank}(S-X+Y+Z)+\operatorname{rank}(S+Y) \geq \operatorname{rank}(S-X+Y)+\operatorname{rank}(S+Y+Z) .
$$

Now, $\operatorname{rank}(S+Y)=|S|$ (given as (c)), $\operatorname{rank}(S-X+Y)=|S|-|X|+|Y|$ (given as (b)), and $\operatorname{rank}(S+Y+Z) \geq \operatorname{rank}(S+Z)=|S|+|Z|$. Thus, $\operatorname{rank}(S-X+Y+Z)=|S|-|X|+|Y|+|Z|$.

\subsection{Augmenting Sets}

Let $S$ be a common independent set in $\mathcal{I}_{1} \cap \mathcal{I}_{2}$. Recall the exchange graph $G(S)$ with respect to this common set $S$, whose vertex set $V(G(S))=V \cup\{s, t\}$. Also recall that $D_{i}$ is the set of elements of $V$ which are at distance exactly $i$ from the source $s \in G(S)$. Next, we define the notion of augmenting sets in the graph $G(S)$. Let $2(\ell+1)$ be the length of the shortest path from $s$ to $t$ in the augmenting graph. We define augmenting sets with respect to this graph $G(S)$.

Definition 24 (Augmenting Sets). Let $S \in \mathcal{I}_{1} \cap \mathcal{I}_{2}$ and $G(S)$ be the corresponding exchange graph with shortest path $2(\ell+1)$. A collection of sets $\Pi_{\ell}:=\left(B_{1}, A_{1}, B_{2}, A_{2}, \ldots, A_{\ell}, B_{\ell+1}\right)$ form an augmenting collection of sets, or simply augmenting sets, in $G(S)$ if the following conditions are satisfied:

(a) For $1 \leq k \leq \ell+1$, we have $A_{k} \subseteq D_{2 k}$ and $B_{k} \subseteq D_{2 k-1}$.

(b) $\left|B_{1}\right|=\left|A_{1}\right|=\left|B_{2}\right|=\cdots=\left|B_{\ell+1}\right|=w$

(c) $S+B_{1} \in \mathcal{I}_{1}$

(d) $S+B_{\ell+1} \in \mathcal{I}_{2}$

(e) For all $1 \leq k \leq \ell$, we have $S-A_{k}+B_{k+1} \in \mathcal{I}_{1}$

(f) For all $1 \leq k \leq \ell$, we have $S-A_{k}+B_{k} \in \mathcal{I}_{2}$

See Figure 6.1 for an illustration. We let $w$ denote the width of $\Pi_{\ell}$.

There is some redundancy in the above definition. For instance, $S+B_{1} \in \mathcal{I}_{1}$ does imply $B_{1} \subseteq D_{1}$, the set of free vertices for $S$ in $\mathcal{I}_{1}$. Nevertheless, we include this in the definition for better understanding. Observe that if each $\left|A_{k}\right|$ was of size 1 , an augmenting set corresponds to an augmenting path. Thus, the concept generalizes the concept of augmenting paths in the exchange graph and in the next subsection we prove many analogous properties.

\subsubsection{Properties of augmenting sets}

Our first goal is to show that given an augmenting set $\Pi_{\ell}$, we can do the natural swap operation to obtain a larger common independent set.

Theorem 25. Let $\Pi_{\ell}:=\left(B_{1}, A_{1}, B_{2}, A_{2}, \cdots, B_{\ell}, A_{\ell}, B_{\ell+1}\right)$ be the an augmenting set in the exchange graph $G(S)$ whose shortest path length is $2(\ell+1)$. Then the set $S^{\prime}:=S \oplus \Pi_{\ell}:=S+B_{1}-$ $A_{1}+B_{2}-\cdots+B_{\ell}-A_{\ell}+B_{\ell+1}$ is a common independent set. 


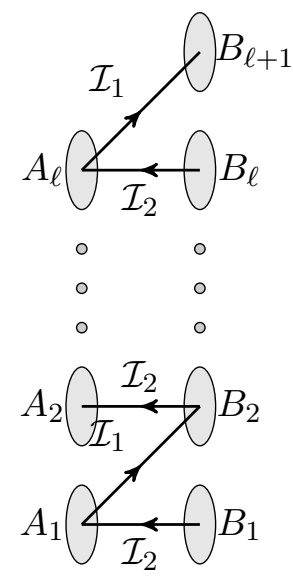

Figure 6.1: Augmenting sets where each $A_{k} \subseteq S$ and $B_{k} \subseteq \bar{S}$. They satisfy $S-A_{k}+B_{k+1} \in \mathcal{I}_{1}$ and $S-A_{k}+B_{k} \in \mathcal{I}_{2}$.

Proof. Let us prove that $S^{\prime} \in \mathcal{I}_{1}$, and the proof of $S^{\prime} \in \mathcal{I}_{2}$ is analogous and omitted.

The first observation is that there is no edge from a vertex $a \in A_{i}$ to a vertex $b \in B_{j}$ with $j>i+1$. The reason is that $A_{i} \subseteq D_{2 i}$ and $B_{j} \subseteq D_{2 j-1}$. If $j>i+1$, then $2 j-1>2 i+1$, and since the $D_{i}$ 's are vertices at distance exactly $i$, there can't be an edge from any vertex in $D_{2 i}$ to any vertex in $D_{2 j-1}$.

First, in the following lemma, we prove $S^{\prime}-B_{1}$ is independent in $\mathcal{M}_{1}$.

Lemma 26. The set $S^{\prime}-B_{1}:=S-A_{1}+B_{2}-A_{2}+\cdots+B_{\ell+1} \in \mathcal{I}_{1}$.

Proof. For brevity, let us use $\mathcal{A}_{k}$ to denote the union of the sets $A_{1}+A_{2}+\cdots+A_{k}$ and let $\mathcal{B}_{k+1}=B_{2}+B_{3}+\cdots+B_{k+1}$. Since all the sets are of equal size, we have $\left|\mathcal{A}_{k}\right|=\left|\mathcal{B}_{k+1}\right|$.

The lemma asks us to prove $S-\mathcal{A}_{\ell}+\mathcal{B}_{\ell+1} \in \mathcal{I}_{1}$. The proof is by induction on $k$. Note that $S-\mathcal{A}_{1}+\mathcal{B}_{2}=S-A_{1}+B_{2} \in \mathcal{I}_{1}$ by the definition of augmenting sets.

Suppose we have already established $X:=S-\mathcal{A}_{k}+\mathcal{B}_{k+1} \in \mathcal{I}_{1}$ and we wish to show $S-\mathcal{A}_{k+1}+$ $\mathcal{B}_{k+2} \in \mathcal{I}_{1}$. From definition we know that $S-A_{k+1}+B_{k+2} \in \mathcal{I}_{1}$. So, $Y:=S-A_{k+1}+B_{k+2}-\mathcal{A}_{k}$ is also in $\mathcal{I}_{1}$. Using this definition, we want to show $Y+\mathcal{B}_{k+1} \in \mathcal{I}_{1}$.

Now, $|X|=|S|$ while $|Y|=|S|-\left|\mathcal{A}_{k}\right|$. Therefore, using the exchange principle of matroids on $X$ and $Y$, we assert that there exists a set

$$
R \subseteq A_{k+1} \cup \mathcal{B}_{k+1}, \quad|R|=\left|\mathcal{A}_{k}\right|=\left|\mathcal{B}_{k+1}\right|, \quad \text { s.t. } \quad Z:=Y+R \in \mathcal{I}_{1}
$$

If $R=\mathcal{B}_{k+1}$, we are done. Therefore suppose $R \neq \mathcal{B}_{k+1}$. In particular, $\left|R \cap \mathcal{B}_{k+1}\right|<\left|\mathcal{B}_{k+1}\right|$. Below we show this leads to a contradiction, completing the proof of the lemma.

Since $Z \in \mathcal{I}_{1}$ with $|Z|=|S|$ and $S-\mathcal{A}_{k} \in \mathcal{I}_{1}$, we know there exists a subset $Q \subseteq Z \backslash\left(S-\mathcal{A}_{k}\right)$ such that $|Q|=\left|\mathcal{A}_{k}\right|$ and $S-\mathcal{A}_{k}+Q \in \mathcal{I}_{1}$. Staring at $Z \backslash\left(S-\mathcal{A}_{k}\right)$, we get that $Q \subseteq\left(R \cap \mathcal{B}_{k+1}\right) \cup B_{k+2}$.

Now, we use the fact that there is no edge in $G(S)$ from $a \in \mathcal{A}_{k}$ to $B_{k+2}$. Using Fact 22 , we get that $S-\mathcal{A}_{k}+b \notin \mathcal{I}_{1}$ for any $b \in B_{k+2}$. That is, the set $Q$ defined above cannot intersect $B_{k+1}$ and thus must be a subset of $R \cap \mathcal{B}_{k+1}$ itself. Since $|Q|=\left|\mathcal{A}_{k}\right|=\left|\mathcal{B}_{k+1}\right|$, we get $\left|R \cap \mathcal{B}_{k+1}\right| \geq\left|\mathcal{B}_{k+1}\right|$ which leads to the promised contradiction.

The theorem now follows from the above lemma. Indeed, the sets $B_{2}, B_{3}, \ldots, B_{\ell+1}$, and in particular, $B_{2}+\cdots+B_{\ell+1}$, all lie in $\operatorname{span}(S)$ in $\mathcal{M}_{1}$. Otherwise, they would lie in the set $D_{1}$ (by definition of the exchange graph). Thus, $S+B_{1} \in \mathcal{I}_{1}$, and from the following lemma $S^{\prime}-B_{1}=$ $S-\left(A_{1}+A_{2}+\ldots+A_{\ell}\right)+\left(B_{2}+\cdots+B_{\ell+1}\right) \in \mathcal{I}_{1}$. The theorem follows from Fact 23. 
In the remainder of the section, we want to show an equivalence between the family of augmentation sets in $G(S)$, and a collection of consecutive shortest augmenting paths in $G(S)$. At a high-level, this equivalence allows us to run a single phase of Cunningham in "one-shot". The following lemma is the main structural fact.

Lemma 27. Let $S$ be a common subset of $\mathcal{I}$ and let $G(S)$ be the corresponding exchange graph. Let $X_{1}, X_{2}, \ldots, X_{k}$ be disjoint subsets of $S$, and let $Y_{1}, Y_{2}, \ldots, Y_{k}$ be disjoint subsets of $\bar{S}$ satisfying $\left|X_{i}\right|=\left|Y_{i}\right|$ for all $1 \leq i \leq k$.

If (a) there is no edge from a vertex in $X_{i}$ to a vertex in $Y_{j}$ with $j>i$, and (b) $S-X_{1}+Y_{1}-$ $X_{2}+Y_{2} \cdots-X_{k}+Y_{k} \in \mathcal{I}_{1}$, then each set $S-X_{i}+Y_{i} \in \mathcal{I}_{1}$.

Similarly, if (a) there is no edge from a vertex in $Y_{j}$ to a vertex in $X_{i}$ with $i>j$, and (b) $S-X_{1}+Y_{1}-X_{2}+Y_{2} \cdots-X_{k}+Y_{k} \in \mathcal{I}_{2}$, then each set $S-X_{i}+Y_{i} \in \mathcal{I}_{2}$.

Proof. We prove the statement for $\mathcal{I}_{1}$; the proof for $\mathcal{I}_{2}$ is analogous.

The base case of $k=2$ has the main ideas and let us first focus on this. Let $S^{\prime}:=S-X_{1}+$ $Y_{1}-X_{2}+Y_{2} \in \mathcal{I}_{1}$. Let $T=S-X_{1} \in \mathcal{I}_{1}$. Using the exchange property, we know there exists $Q \subseteq Y_{1}+Y_{2}$ of size $|Q|=\left|X_{1}\right|=\left|Y_{1}\right|$, such that $S-X_{1}+Q \in \mathcal{I}_{1}$. Since there is no edge from any vertex in $X_{1}$ to any vertex in $Y_{2}$, using Fact 22 we know that $S-X_{1}+y \notin \mathcal{I}_{1}$ for any $y \in Y_{2}$. Thus, $Q \subseteq Y_{1}$. Since $|Q|=\left|Y_{1}\right|$, we get $Q=Y_{1}$. That is, $S-X_{1}+Y_{1} \in \mathcal{I}_{1}$.

For the second set, we need to work a bit more. We know that $S-X_{1}-X_{2}+Y_{2} \in \mathcal{I}_{1}$ and $S \in \mathcal{I}_{1}$. Thus, there exists a set $R \subseteq X_{1}+X_{2}$ such that $|R|=\left|X_{1}\right|$ and $S-X_{1}-X_{2}+Y_{2}+R \in \mathcal{I}_{1}$. If $R=X_{1}$, we are done. If $R \neq X_{1}$, then since $|R|=\left|X_{1}\right|$, there must exist an $x \in X_{2} \cap R$. That is, the set $T:=S-X_{1}-X_{2}+Y_{2}+x \in \mathcal{I}$. Note $|T|=|S|-\left|X_{1}\right|+1$. Applying the exchange property with the independent set $S-X_{1}$, we get the existence of $y \in T \backslash\left(S-X_{1}\right)$ such that $S-X_{1}+y \in \mathcal{I}$. Since $X_{2} \subseteq\left(S-X_{1}\right)$, we get that $y \neq x$. In particular, $y \in Y_{2}$. But, as established before, $S-X_{1}+y \notin \mathcal{I}$ for all $y \in Y_{2}$ (since there is no edge from $X_{1}$ to $Y_{2}$ ).

Now we handle the general case $k>2$ by applying the result for $k=2$ twice. By considering $X_{1}^{\prime}=X_{1}+\ldots+X_{i}, X_{2}^{\prime}=X_{i+1}+\ldots+X_{k}$ (and set $Y_{1}^{\prime}, Y_{2}^{\prime}$ similarly) we have $S-X_{1}^{\prime}+Y_{1}^{\prime} \in \mathcal{I}_{1}$. Finally, by considering $X_{1}^{\prime \prime}=X_{1}^{\prime}-X_{i}, X_{2}^{\prime \prime}=X_{i}$ we have $S-X_{i}+Y_{i} \in \mathcal{I}_{1}$.

Next, we define the notion of consecutive shortest augmenting paths. Again, each phase of Cunningham's algorithm finds such an ordered collection.

Definition 28. Let $S$ be a common independent set in $\mathcal{I}_{1} \cap \mathcal{I}_{2}$. Let $G(S)$ be the exchange graph with shortest path $2(\ell+1)$. Let $\mathcal{P}=\left(p_{1}, p_{2}, \ldots, p_{k}\right)$ be an ordered collection of consective shortest augmenting paths if (a) each $p_{i}$ is of length $2(\ell+1)$, and (b) $p_{i}$ is a valid path in the exchange graph $G\left(S_{i}\right)$ where $S_{i}$ is obtained after augmenting along the paths $p_{1}, \ldots, p_{i-1}$ in that order.

Theorem 29. Let $S$ be a common independent set in $\mathcal{I}_{1} \cap \mathcal{I}_{2}$. Given an ordered collection $\mathcal{P}$ of consecutive shortest augmenting paths in $G(S)$, we can find an augmenting set $\Pi_{\ell}$ of width $|\mathcal{P}|$.

Proof. This follows almost immediately from Lemma 27. Let $|\mathcal{P}|=w$. Let the path $p_{i}:=\left(s, b_{1}^{(i)}, a_{1}^{(i)}, b_{2}^{(i)}, \cdots, b_{\ell}^{(i)}, a_{\ell}^{(i)}, b_{\ell+1}^{(i)}, t\right)$ where $b_{k}^{(i)} \in D_{2 k-1}$ and $a_{k}^{(i)} \in D_{2 k}$.

Define $X_{k}:=\left\{a_{k}^{(1)}, a_{k}^{(2)}, \cdots, a_{k}^{(w)}\right\}$ and $Y_{k}=\left\{b_{k+1}^{(1)}, b_{k+1}^{(2)}, \cdots, b_{k+1}^{(w)}\right\}$. Since augmenting consecutive shortest paths preserve independence, and since augmentations don't introduce new shortcuts by the monotonicity lemma (Lemma 9), we see that the sets satisfy the conditions of Lemma 27 with $\mathcal{I}=\mathcal{I}_{1}$.

Similary, for $\mathcal{I}_{2}$ we consider $X_{k}:=\left\{a_{k}^{(1)}, a_{k}^{(2)}, \cdots, a_{k}^{(w)}\right\}$ and $Y_{k}=\left\{b_{k}^{(1)}, b_{k}^{(2)}, \cdots, b_{k}^{(w)}\right\}$ instead. 
Next, we show that any augmenting set of width $w$ can be peeled out into a sequence of consecutive shortest augmenting paths. We start with a few definitions.

Definition 30. Let $\Pi_{\ell}:=\left(B_{1}, A_{1}, B_{2}, \cdots, B_{\ell}, A_{\ell}, B_{\ell+1}\right)$ be an augmenting set in $G(S)$. Let $S^{\prime}=$ $S \oplus \Pi_{\ell}$ be as defined in Theorem 25. We denote the exchange graph of $G\left(S^{\prime}\right)$ as $G(S) \mid \Pi_{\ell}$.

Definition 31. Let $S$ be a common independent set and $G(S)$ be the exchange graph with shortest path $2(\ell+1)$. Let $\Pi_{\ell}=\left(B_{1}, A_{1}, B_{2}, \cdots, B_{\ell}, A_{\ell}, B_{\ell+1}\right)$ and $\widetilde{\Pi}_{\ell}=\left(\widetilde{B}_{1}, \widetilde{A}_{1}, \widetilde{B}_{2}, \cdots, \widetilde{B}_{\ell}, \widetilde{A}_{\ell}, \widetilde{B}_{\ell+1}\right)$ be two augmeting sets in $G(S)$. We say $\widetilde{\Pi}_{\ell}$ contains $\Pi_{\ell}$ if $B_{k} \subseteq \widetilde{B}_{k}$ and $A_{k} \subseteq \widetilde{A}_{k}$, for all $k$. We use the notation $\Pi_{\ell} \subseteq \widetilde{\Pi}_{\ell}$ to denote this.

Definition 32. Let $S$ be a common independent set and $G(S)$ be the exchange graph with shortest path $2(\ell+1)$. Let $\Pi_{\ell} \subseteq \widetilde{\Pi}_{\ell}$ be two augmenting sets, the former contained in the latter. Then we use $\widetilde{\Pi}_{\ell} \backslash \Pi_{\ell}$ to denote the sequence $\left(\widetilde{B}_{1} \backslash B_{1}, \widetilde{A}_{1} \backslash A_{1}, \cdots, \widetilde{B}_{\ell+1} \backslash B_{\ell+1}\right)$.

Theorem 33. Let $\Pi_{\ell} \subseteq \widetilde{\Pi}_{\ell}$ be two augmenting sets in $G(S)$. Then, $\widetilde{\Pi}_{\ell} \backslash \Pi_{\ell}$ is an augmenting set in $G(S) \mid \Pi_{\ell}$.

Proof. Let $P_{k}:=\widetilde{A}_{k} \backslash A_{k}$ and let $Q_{k}=\widetilde{B}_{k} \backslash B_{k}$. Let $S^{\prime}=S+B_{1}-A_{1}+B_{2}-A_{2}+\cdots+B_{\ell+1}$. Note that this is $S \oplus \Pi_{\ell}$. For brevity, let $G^{\prime}:=G\left(S^{\prime}\right)=G(S) \mid \Pi_{\ell}$. Let the layers of $G^{\prime}$ be denoted as $D_{1}^{\prime}, D_{2}^{\prime}, \ldots$ Furthermore, since $\widetilde{\Pi}_{\ell}$ is an augmenting set, we get that

$$
S^{\prime}+Q_{1}-P_{1}+Q_{2}+\cdots+Q_{\ell+1} \in \mathcal{I}_{1} \cap \mathcal{I}_{2}
$$

as well.

Using the above, we can show that each $P_{k} \subseteq D_{2 k}^{\prime}$ and $Q_{k} \subseteq D_{2 k-1}^{\prime}$. For instance, we can show $S^{\prime}+Q_{1} \in \mathcal{I}_{1}$ (which would put $Q_{1} \subseteq D_{2 k-1}^{\prime}$ ). To see this, let $\mathcal{P}:=P_{1}+\cdots+P_{\ell}$ and $\mathcal{Q}=Q_{2}+\cdots+Q_{\ell+1}$. Note, $|\mathcal{P}|=|\mathcal{Q}|$ and $S^{\prime}+Q_{1}-\mathcal{P}+\mathcal{Q} \in \mathcal{I}_{1}$. Now observe $\mathcal{Q} \cap D_{1}^{\prime}=\emptyset$; this follows from Cunningham's monotonicity lemma (Lemma 9). Thus, $\mathcal{Q} \in \operatorname{span}_{1}\left(S^{\prime}\right)$. Thus, the independence of $S+Q_{1}-\mathcal{P}+\mathcal{Q}$ implies $^{4} S+Q_{1} \in \mathcal{I}_{1}$. For the general case one proceeds inductively. This proves condition (a) of the augmenting set definition.

Condition (b) holds since $\widetilde{\Pi}$ and $\Pi$ were valid augmenting sets. Condition (c),(d) is proved as above (the base case was (c)).

Finally, condition (e) and (f) follow from Lemma 27. To see this, we need the observation that there is no edge from $P_{i}$ to $Q_{j}$ for $j>i+1$ and from $Q_{j}$ to $P_{i}$ for $i>j$, in $G^{\prime}$. To see this, this was true in $G(S)$ (shortest path property). And since the levels do not change, and the monotonicity lemma (Lemma 9) this continues to hold. Now Lemma 27 (with $X_{i}$ 's and $Y_{i}$ 's properly defined) implies conditions (e) and (f).

Thus, $\widetilde{\Pi}_{\ell} \backslash \Pi_{\ell}$ is a valid augmenting set in $G^{\prime}=G(S) \mid \Pi_{\ell}$.

Now we are armed to prove the following theorem.

Theorem 34. Let $S \in \mathcal{I}_{1} \cap \mathcal{I}_{2}$ and $G(S)$ be the exchange graph. Let $\Pi_{\ell}$ be an augmenting set of width $w$. Then there exists an ordered collection $\mathcal{P}$ of $w$ consecutive shortest augmenting paths.

Proof. We first show there is one shortest augmenting path hitting every set of $\Pi_{\ell}$ exactly once. We augment on it and apply Theorem 33.

Let $\Pi_{\ell}=\left(B_{1}, A_{1}, B_{2}, \ldots, B_{\ell}, A_{\ell}, B_{\ell+1}\right)$. We claim there is a path $p=\left(b_{1}, a_{1}, b_{2}, \ldots, b_{\ell}, a_{\ell}, b_{\ell+1}\right)$ such that $b_{k} \in A_{k}$ and $a_{k} \in A_{k}$. Indeed, to prove this it suffice to show that

$$
{ }^{4}|S|+\left|Q_{1}\right|=\operatorname{rank}_{1}\left(S+Q_{1}-\mathcal{P}+\mathcal{Q}\right) \leq \operatorname{rank}_{1}\left(S+Q_{1}+\mathcal{Q}\right) \leq \operatorname{rank}_{1}\left(S+Q_{1}\right)
$$


(i) for $k=1, \ldots, \ell$, for any $b \in B_{k}$, there is an edge $(b, a) \in G(S)$ to some $a \in A_{k}$

(ii) for $k=1, \ldots, \ell$, for any $a \in A_{k}$, there is an edge $(a, b) \in G(S)$ to some $b \in B_{k+1}$.

For (i), notice that $S-A_{k}+B_{k} \in \mathcal{I}_{2}$ (condition (f) of augmenting set), and so $S-A_{k}+b \in \mathcal{I}_{2}$. The contrapositive of Fact 22 implies there must be an edge from $b$ to some vertex in $A_{k}$.

For (ii) notice that since $S-A_{k}+B_{k+1} \in \mathcal{I}_{1}$ (condition (e) of augmenting set), for any $a \in A_{k}$, we have $\operatorname{rank}_{1}\left(S-a+B_{k+1}\right) \geq \operatorname{rank}_{1}\left(S-A_{k}+B_{k+1}\right)=|S|$ since $\left|A_{k}\right|=\left|B_{k+1}\right|$. Since $\operatorname{rank}_{1}(S-a)=|S|-1$, by exchange property of matroids there must exist $b \in B_{k+1}$ such that $S-a+b \in \mathcal{I}_{1}$. That is, $(a, b) \in G(S)$.

To complete the proof of the theorem, we observe that augmenting paths are width-1 augmenting sets. We apply Theorem 33 to get $w$ consecutive paths.

For our algorithms we will not be interested in finding arbitrary augmenting sets, but in finding maximal augmenting sets.

Definition 35 (Maximal Augmenting Sets). Let $S \in \mathcal{I}_{1} \cap \mathcal{I}_{2}$ and let $G(S)$ be the corresponding exchange graph with shortest augmenting path of length $2(\ell+1)$. An augmenting set $\Pi_{\ell}$ is called maximal if there exists no other augmenting set $\widetilde{\Pi}_{\ell}$ containing $\Pi_{\ell}$.

Theorem 36. Let $S \in \mathcal{I}_{1} \cap \mathcal{I}_{2}$ and let $G(S)$ be the corresponding exchange graph with shortest augmenting path of length $2(\ell+1)$. Let $\Pi_{\ell}$ be a maximal augmenting set. Then there is no augmenting path of length $2(\ell+1)$ in $G(S) \mid \Pi_{\ell}$.

Proof. This is a corollary of Theorem 29 and Theorem 34. Let $\mathcal{P}$ be the collection of paths obtained by invoking Theorem 34 on $\Pi_{\ell}$. If there is another shortest augmenting path $p \in G(S) \mid \Pi_{\ell}$, then $(\mathcal{P}, p)$ is an ordered collection of consecutive shortest augmenting paths in $G(S)$. Thus Theorem 29 invoked on this returns $\widetilde{\Pi}_{\ell}$ which contains $\Pi_{\ell}$.

The above lemma shows that a single phase of Cunningham's algorithm corresponds to finding maximal augmenting sets. This is what we do in Section 6.3, but we need one more concept before: partial augmenting sets.

\subsubsection{Partial Augmenting Sets}

In order to find maximal augmenting sets, our algorithm will leverage objects which are not augmenting sets at all. These are what we call partial augmenting sets, and the final algorithm will work work by incrementally finding better partial augmenting sets, from which augmenting sets can be extracted.

Definition 37 (Partial Augmenting Sets). Let $S \in \mathcal{I}_{1} \cap \mathcal{I}_{2}$ and $G(S)$ be the corresponding exchange graph with shortest path $2(\ell+1)$. A collection of sets $\Phi_{\ell}:=\left(B_{1}, A_{1}, B_{2}, A_{2}, \ldots, A_{\ell}, B_{\ell+1}\right)$ form a partial augmenting set if the following conditions are satisfied.

(a) For $1 \leq k \leq \ell+1$, we have $A_{k} \subseteq D_{2 k}$ and $B_{k} \subseteq D_{2 k-1}$.

(b) $\left|B_{1}\right| \geq\left|A_{1}\right| \geq\left|B_{2}\right| \geq \cdots \geq\left|B_{\ell+1}\right|$.

(c) $S+B_{1} \in \mathcal{I}_{1}$

(d) $S+B_{\ell+1} \in \mathcal{I}_{2}$

(e) For all $1 \leq k \leq \ell$, we have $S-A_{k}+B_{k+1} \in \mathcal{I}_{1}$.

(f) For all $1 \leq k \leq \ell$, we have $\operatorname{rank}_{2}\left(S-A_{k}+B_{k}\right)=\operatorname{rank}_{2}(S)$. 
As in Definition 31, we say $\Pi_{\ell} \subseteq \Phi_{\ell}$ if each "coordinate" of $\Pi_{\ell}$ is a subset of the corresponding "coordinate" of $\Phi_{\ell}$. The following lemma gives an efficient algorithm to convert any partial augmenting set $\Phi_{\ell}$ into a true augmenting set $\Pi_{\ell}$. For now, the reader should consider $\Pi_{\ell}^{\prime}$ to be a string of empty sets; we will invoke this lemma later with non-empty $\Pi_{\ell}^{\prime}$.

Lemma 38. Given a partial augmenting set $\Phi_{\ell}=\left(B_{1}, A_{1}, B_{2}, \cdots, B_{\ell}, A_{\ell}, B_{\ell+1}\right)$ that contains an augmenting set $\Pi_{\ell}^{\prime}=\left(B_{1}^{\prime}, A_{1}^{\prime}, \ldots, B_{\ell}^{\prime}\right)$, in $O\left(n \cdot \mathcal{T}_{\text {ind }}\right)$-time we can find an augmenting set $\widetilde{\Pi}_{\ell}=$ $\left(\widetilde{B}_{1}, \widetilde{A}_{1}, \widetilde{B}_{2}, \cdots, \widetilde{B}_{\ell}, \widetilde{A}_{\ell}, \widetilde{B}_{\ell+1}\right)$ such that

(a) $\Pi_{\ell}^{\prime} \subseteq \widetilde{\Pi}_{\ell} \subseteq \Phi_{\ell}$, and

(b) $\widetilde{B}_{\ell+1}=B_{\ell+1}$.

Proof. We work backwards from $\widetilde{B}_{\ell+1}=B_{\ell+1}$. Let $w=\left|B_{\ell+1}\right|$. Suppose we have constructed sets $\widetilde{B}_{\ell+1}, \widetilde{A}_{\ell}, \cdots, \widetilde{B}_{k+1}$. Now, since $S-A_{k}+\widetilde{B}_{k+1} \subseteq S-A_{k}+B_{k+1}$, and since the latter lies in $\mathcal{I}_{1}$ (property (e) of partial augmenting sets), we have $S-A_{k}+\widetilde{B}_{k+1} \in \mathcal{I}_{1}$. We also have $S-A_{k}^{\prime}+B_{k}^{\prime} \in \mathcal{I}_{1}$. Therefore, by the exchange property, we can find a set $\widetilde{A}_{k}$ of size $\left|\widetilde{A}_{k}\right|=\left|\widetilde{B}_{k+1}\right|=w$ such that $A_{k}^{\prime} \subseteq \widetilde{A}_{k} \subseteq A_{k}$ and $S-\widetilde{A}_{k}+\widetilde{B}_{k+1} \in \mathcal{I}_{1}$. The time taken to do so is $O\left(\left|A_{k}\right|\right)$ independence-query oracles.

Now, suppose we have constructed $\widetilde{B}_{\ell+1}, \widetilde{A}_{\ell}, \cdots, \widetilde{A}_{k}$ with the desired property. Then since $\widetilde{A}_{k} \subseteq A_{k}$ and $\operatorname{rank}_{2}\left(S-A_{k}+B_{k}\right)=\operatorname{rank}_{2}(S)$, we get $\operatorname{rank}_{2}\left(S-\widetilde{A}_{k}+B_{k}\right) \geq \operatorname{rank}_{2}(S)$. Also, since $S-A_{k}^{\prime}+B_{k}^{\prime} \in \mathcal{I}_{2}$, we get $S-\widetilde{A}_{k}+B_{k}^{\prime} \in \mathcal{I}_{2}$ as well. Thus, we can select a subset $\widetilde{B}_{k}$ such that $B_{k}^{\prime} \subseteq B_{k} \subseteq B_{k}$ and $S-\widetilde{A}_{k}+\widetilde{B}_{k} \in \mathcal{I}_{2}$ and has size $|S|$. That is, $\left|\widetilde{B}_{k}\right|=\left|\widetilde{A}_{k}\right|=w$. The time taken to find this is $O\left(\left|B_{k}\right|\right)$ independence oracle queries.

The total running time is $\sum_{k} O\left(\left|A_{k}\right|+\left|B_{k}\right|\right)$ many independence oracle calls which is $O\left(n \cdot \mathcal{T}_{\text {ind }}\right)$ time.

Now we are ready to prove a key property which relates the width of any two maximal augmenting sets. This will be crucial to give the subquadratic approximation algorithm in Section 6.4.

Lemma 39. Let $S \in \mathcal{I}_{1} \cap \mathcal{I}_{2}$ and $G(S)$ be the corresponding graph with shortest augmenting path of length $2(\ell+1)$. Let $\Pi_{\ell}$ and $\widetilde{\Pi}_{\ell}$ be two maximal augmenting sets of width $w$ and $\widetilde{w}$. Then, $\widetilde{w} \leq(2 \ell+4) w$.

Proof. Let $\Pi_{\ell}=\left(B_{1}, A_{1}, B_{2}, \ldots, A_{\ell}, B_{\ell+1}\right)$. Let $\widetilde{\Pi}_{\ell}=\left(Q_{1}, P_{1}, Q_{2}, \ldots, P_{\ell}, Q_{\ell+1}\right)$. Note $\left|A_{k}\right|=$ $\left|B_{j}\right|=w$ and $\left|P_{k}\right|=\left|Q_{j}\right|=\widetilde{w}$. For the sake of contradiction, assume $\widetilde{w}>(2 \ell+4) w$.

We construct partial augmenting sets $\Phi_{\ell}=\left(B_{1}^{\prime}, A_{1}^{\prime}, \cdots, A_{\ell}^{\prime}, B_{\ell+1}^{\prime}\right)$ such that (i) $A_{k} \subseteq A_{k}^{\prime} \subseteq$ $A_{k}+P_{k}$, (ii) $B_{k} \subseteq B_{k}^{\prime} \subseteq B_{k}+Q_{k}$, (iii) $\left|A_{k}^{\prime}\right|>(2 \ell+3-2 k) \cdot\left|A_{k}\right|$, and (iv) $\left|B_{k}^{\prime}\right|>(2 \ell+4-2 k) \cdot\left|B_{k}\right|$, and $(\mathrm{v})\left|B_{\ell+1}^{\prime}\right|>\left|B_{\ell+1}\right|$. Note that the last point would imply $B_{\ell+1}$ is a strict subset of $B_{\ell+1}^{\prime}$. From Lemma 38 , we would get an augmenting set $\Pi_{\ell}^{\prime}$ which would contain $\Pi_{\ell}$, contradicting the maximality of the latter.

Now we describe the construction of $\Phi_{\ell}$.

To construct $B_{1}^{\prime}$, we start with $B_{1}$ and keep adding elements of $Q_{1}$ to it maintaining $S+B_{1}^{\prime} \in \mathcal{I}_{1}$. By the matroid exchange property, we will add $\left|Q_{1} \backslash B_{1}\right|$ such elements giving $\left|B_{1}^{\prime}\right|=\left|Q_{1}\right|=\widetilde{w}>$ $(2 \ell+4) w$. We satisfy (i), (iv), and property (c) of the partial augmenting set.

Suppose we have constructed $B_{1}^{\prime}, A_{1}^{\prime}, B_{2}^{\prime}, \ldots, B_{k}^{\prime}$ as desired. We need to construct $A_{k}^{\prime}$. Since $S-A_{k}+B_{k} \in \mathcal{I}_{2}$, we get $S-\left(P_{k}+A_{k}\right)+B_{k} \in \mathcal{I}_{2}$. We also have $S-P_{k}+Q_{k} \in \mathcal{I}_{2}$. As $B_{k}^{\prime} \subseteq B_{k}+Q_{k}$, we get that $S-P_{k}+\left(B_{k}^{\prime} \backslash B_{k}\right) \in \mathcal{I}_{2}$. By the exchange property, there must exist a $Z \subseteq B_{k}^{\prime} \backslash B_{k}$ such that $S-\left(P_{k}+A_{k}\right)+B_{k}+Z \in \mathcal{I}_{2}$ and

$$
\left|B_{k}+Z\right|=\left|B_{k}^{\prime} \backslash B_{k}\right| \geq\left|B_{k}^{\prime}\right|-\left|B_{k}\right|>(2 \ell+4-2 k)\left|B_{k}\right|-\left|B_{k}\right|=(2 \ell+3-2 k) w
$$


where the inequality follows from (iv) above (which we assume we have maintained so far). Now since $S-A_{k}+B_{k} \in \mathcal{I}_{2}$, there is some $A_{k}^{\prime}$ of size $\left|B_{k}+Z\right|$ s.t. $A_{k} \subseteq A_{k}^{\prime} \subseteq A_{k}+P_{k+1}$ and $\operatorname{rank}_{2}\left(S-A_{k}^{\prime}+B_{k}+Z\right)=\operatorname{rank}_{2}(S)$. Thus, property (d) of the partial augmenting set is satisfied, and so is property (iii).

Now, suppose we have constructed $B_{1}^{\prime}, A_{1}^{\prime}, B_{2}^{\prime}, \ldots, A_{k}^{\prime}$ as desired. We need to construct $B_{k+1}^{\prime}$. As $S-P_{k}+Q_{k+1} \in \mathcal{I}_{1}$, there must exist some $Z \subseteq Q_{k}$ for which $S-A_{k}^{\prime}+Z \in \mathcal{I}_{1}$ and

$$
|Z|=\left|A_{k}^{\prime} \cap P_{k}\right| \geq\left|A_{k}^{\prime}\right|-\left|A_{k}\right|>(2 \ell+3-2 k)\left|A_{k}\right|-\left|A_{k}\right|=(2 \ell+2-2 k) w .
$$

Now consider $S-A_{k}^{\prime}+B_{k+1}$ which is independent since $A_{k} \subseteq A_{k}^{\prime}$. We can then find $B_{k+1}^{\prime}$ of size $|Z|$ s.t. $B_{k+1} \subseteq B_{k+1}^{\prime} \subseteq B_{k+1}+Z$ and $S-A_{k}^{\prime}+B_{k+1}^{\prime} \in \mathcal{I}_{1}$. Note that property (iv) is satisfied for $\left|B_{k+1}^{\prime}\right|$. And the above satisfies property (e) of the partial augmenting sets.

Finally, we need to satisfy condition (c), that is, $S+B_{\ell+1}^{\prime} \in \mathcal{I}_{2}$. After the above procedure, we do get $B_{\ell+1} \subseteq B_{\ell+1}^{\prime} \subseteq B_{\ell+1}+Q_{\ell+1}$ satisfying every property except perhaps this one. Also, $\left|B_{\ell+1}^{\prime}\right|>(2 \ell+4-2(\ell+1)) w=2 w$. Now, since $S+Q_{\ell+1} \in \mathcal{I}_{2}$, we know that $B_{\ell+1}^{\prime} \backslash B_{\ell+1} \in \mathcal{I}_{2}$. Thus, we keep deleting items from $B_{\ell+1}^{\prime}$ such that we get $S+B_{\ell+1}^{\prime} \in \mathcal{I}_{2}$. We will delete at most $\left|B_{\ell+1}\right|=w$ elements. The final $B_{\ell+1}^{\prime}$ will satisfy condition (c) and $\left|B_{\ell+1}^{\prime}\right|>w$, that is, property (v). This completes the proof.

\subsection{The Augmenting Sets Algorithm}

\subsubsection{An Analogy and High level idea}

Before presenting our augmenting sets algorithm, we provide an analogy to finding a maximal collection of disjoint shortest augmenting paths. Recall that the Hopcroft-Karp idea is inapplicable because augmenting such a collection do not necessarily preserve independence. However, let us pretend for the moment that this was indeed our task. Our algorithm in the next section is in fact inspired by this.

Suppose that we have constructed internally disjoint partial augmenting paths from the source. Our goal is to extend them all the way to the sink. Let $A_{k} \subseteq D_{2 k}$ be the sets of vertices on the current augmenting sets in $D_{2 k}$. Define $B_{k}$ similarly. We then have $\left|B_{1}\right| \geq\left|A_{1}\right| \geq \ldots \geq\left|B_{\ell+1}\right|$.

Let us focus on $A_{k}$ and $B_{k+1}$. Because they represent the vertices on partial augmenting paths, currently we have a matching between a subset of $A_{k}$ and $B_{k+1}$. A natural idea is then to match more vertices of $D_{2 k+1}$ with the unmatched vertices of $A_{k}$. Let $B \subseteq D_{2 k+1}$ be such a maximal subset, and $A \subseteq A_{k}$ be the vertices that are still not matched. Such a maximal set requires only $O\left(\left|D_{2 k+1}\right|\right)$ independence oracle calls.

The crucial observation is that all of $A$ 's outgoing edges go to $B_{k}+B$ as $B$ is maximal. However, $B_{k}+B$ is already fully matched; so $A$ is essentially useless as its vertices would never get matched and can be removed from future consideration. It is easy to verify that a total of $\left|B_{k}\right|-\left|A_{k}\right|$ elements are matched or become useless.

A similar operation exists for extending the matching between $B_{k}$ and $A_{k+1}$. By repeating these operations we will eventually find a maximal collection of disjoint shortest augmenting paths. Our treatment of this similar scenario forms the basis of the augmenting sets algorithm given below.

\subsubsection{The Detailed Algorithm}

In this section we describe a (slow) $O\left(n^{2} \cdot \mathcal{T}_{\text {ind }}\right)$-time algorithm to find a maximal augmenting set. It is an iterative algorithm which finds "better" partial augmenting sets in each iteration. The algorithm assumes access to the layers $D_{k}$ 's of the current exchange graph $G(S)$ which has shortest path length $2(\ell+1)$. 
We maintain three types of elements in each layer : selected elements denoted as $A_{k}, B_{k}$, removed elements denoted as $R_{k}$, and fresh $F_{k}$. The selected elements $A_{k} \subseteq D_{2 k}, B_{k} \subseteq D_{2 k-1}$ form a partial augmenting set, and our algorithm incrementally finds better and better partial augmenting sets with larger $B_{\ell+1}$. The removed elements $R_{k} \subseteq D_{k}$ are deemed to be useless for finding better partial augmenting sets. Finally, fresh elements $F_{k} \subseteq D_{k}$ are those that are neither selected nor removed. The type of an element can change from fresh to selected and from selected to removed but never the other way.

Initially, $B_{1}$ is a maximal subset of $\bar{S}$ that can be added to $S$ while being independent in $\mathcal{I}_{1}$. Subsequently, $F_{1}=D_{1} \backslash B_{1}$ and $R_{1}=\emptyset$. For $2 \leq k \leq 2 \ell+1$, we initialize sets $A_{k}, B_{k}, R_{k}=\emptyset$ and $F_{k}=D_{k}$. For convenience we set $A_{0}=R_{0}=F_{0}=D_{0}=\emptyset$ and $A_{\ell+1}=R_{2 \ell+2}=F_{2 \ell+2}=D_{2 \ell+2}=\emptyset$.

We maintain the following invariants. It is easy to verify that the initial conditions satisfy them.

(a) For $1 \leq k \leq \ell$, we have $S-A_{k}+B_{k+1} \in \mathcal{I}_{1}$.

(b) $\operatorname{rank}_{2}\left(S-A_{k}+B_{k}\right)=\operatorname{rank}_{2}(S)$.

Equivalently, $\exists B \subseteq B_{k}$ of size $|B|=\left|A_{k}\right|$ for which $S-A_{k}+B \in \mathcal{I}_{2}$.

(c) For $1 \leq k \leq \ell$, for any $X \subseteq B_{k+1}+F_{2 k+1}=D_{2 k+1}-R_{2 k+1}$, if $S-\left(A_{k}+R_{2 k}\right)+X \in \mathcal{I}_{1}$ then $S-A_{k}+X \in \mathcal{I}_{1}$.

(d) $R_{2 k-1} \in \operatorname{span}_{2}\left(S-\left(D_{2 k}-R_{2 k}\right)+B_{k}\right)$

Invariants (a) and (b) ensure that the selected sets $\Phi_{\ell}:=\left(B_{1}, A_{1}, B_{2}, \cdots, A_{\ell}, B_{\ell+1}\right)$ form a partial augmenting set. Invariant (c) essentially says that if $R_{2 k+1}$ is "useless" (note $X \cap R_{2 k+1}$ is empty), so is $R_{2 k}$. On the other hand, Invariant (d) says that if $R_{2 k}$ is "useless", so is $R_{2 k-1}$. Invariants (c) and (d) are important for certifying that removed elements do not need be considered at all (see Lemma 44).

We are ready to present Refine, which consists of a series of operations on the pairs $\left(A_{k}, B_{k+1}\right)$ and $\left(B_{k}, A_{k}\right)$ inspired by the matching analogy given in Section 6.3.1. For $\left(A_{k}, B_{k+1}\right)$, we extend $B_{k+1}$ as much as possible while respecting Invariant (a) (Lines 1-2 of Refine1 $(k)$ ). We then identify the "matched" vertices in $A_{k}$ and remove the ones still not matched (Lines 3-4 of Refine1 $(k)$ ).

Similarly, for $\left(B_{k}, A_{k}\right)$, we find a maximal subset of $B_{k}$ that can be "matched" and remove the rest (lines 1-2 of Refine2( $k)$ ). We then find the "endpoints" in $A_{k}$ that are "matched" to $B_{k}$ (lines 3-4 of Refine2 $(k))$.

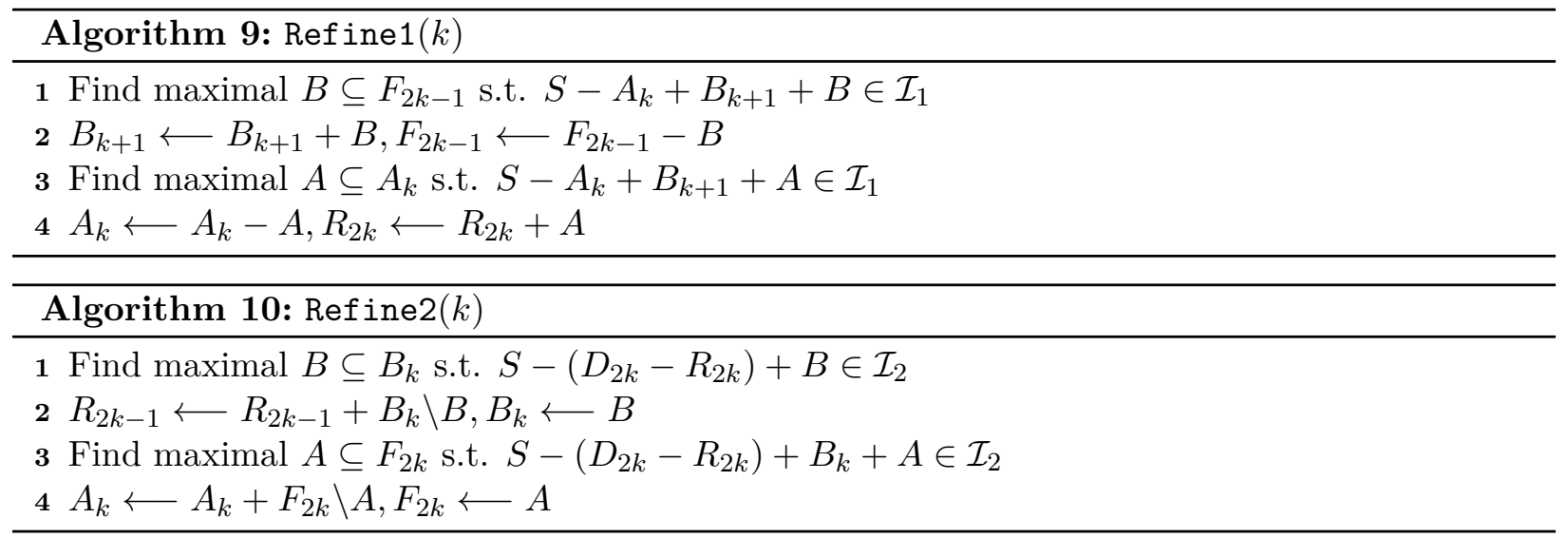




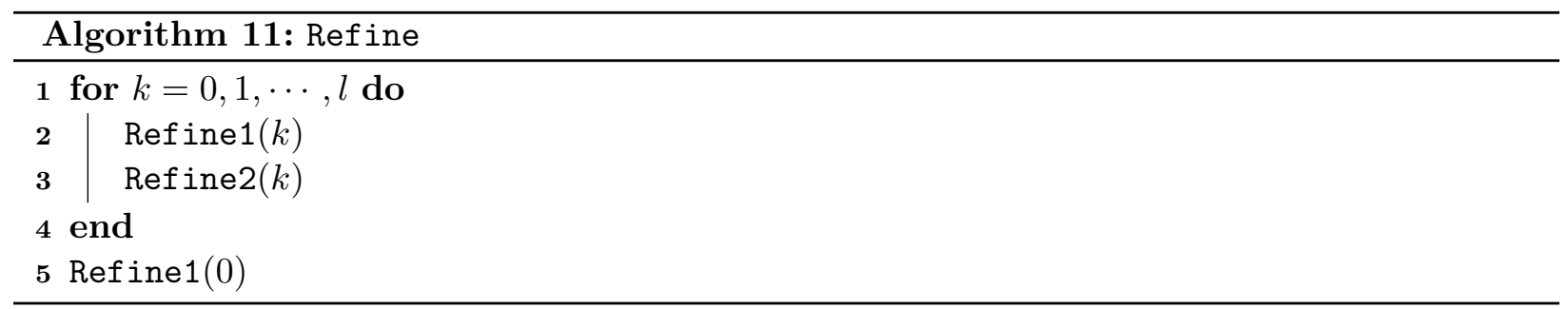

It is somewhat strange that Refine ends with Refine1(0) which is indeed redundant. However it makes the analysis of the combined algorithm in the next subsection less cumbersome (specifically Lemma 47).

The next three lemmas study properties of Refine1 and Refine2 which will be crucial for analyzing the performance of our main subroutine Refine.

Lemma 40. After Refine1( $k)$ is run, we have $\left|A_{k}\right|=\left|B_{k+1}\right|$. After Refine2( $\left.k\right)$ is run, we have $\left|B_{k}\right|=\left|A_{k}\right|$.

Proof. We prove the first statement; the second statement's proof is analogous. Let $A_{k}^{\text {old }}$ and $B_{k+1}^{\text {old }}$ be the sets before Refine $1(k)$ is called. In Line 2 , we set $B_{k+1}$. At this point, let $T:=$ $S-A_{k}^{\text {old }}+B_{k+1}$; we know $T \in \mathcal{I}_{2}$. We also know $\operatorname{rank}_{1}\left(T+A_{k}^{\text {old }}\right)=\operatorname{rank}_{1}\left(S+B_{k+1}\right)=|S|$ since $B_{k+1}$ is in the span of $S$. This implies, the maximal set $A$ added in Line 4 leads to $A_{k}$ such that $\operatorname{rank}_{1}\left(S-A_{k}+B_{k+1}\right)=|S|$, implying $\left|A_{k}\right|=\left|B_{k+1}\right|$.

Lemma 41 (Properties of Refine1). Let $A_{k}^{\text {old }}, B_{k+1}^{\text {old }}$ be the sets $\left(A_{k}, B_{k+1}\right)$ before Refine1( $\left.k\right)$ is called. After the call, all four invariants are preserved. Moreover, for $k \geq 1$ a total of $\left|A_{k}^{\text {old }}\right|-\left|B_{k+1}^{\text {old }}\right|$ elements which were formerly fresh are now selected, or formerly selected but now removed.

Proof. After running Refine1 $(k)$, we have $\left|A_{k}\right|=\left|B_{k+1}\right|$ by Lemma 40. Also note that $\left|B_{k+1}\right|-$ $\left|B_{k+1}^{\text {old }}\right|$ elements are selected and $\left|A_{k}^{\text {old }}\right|-\left|A_{k}\right|$ are removed. Thus a total of $\left|A_{k}^{\text {old }}\right|-\left|B_{k}^{\text {old }}\right|$ elements change status.

Invariant (a) is true by design.

Invariant (b) remains true because $B_{k}$ is unchanged and $A_{k}$ only loses elements.

Invariant (d) remains true because $R_{2 k}$ is only increased, and thus its span can only become bigger.

For Invariant (c), first notice $A_{k}+R_{2 k}=A_{k}^{\text {old }}+R_{2 k}^{\text {old }}$. Since the invariant held before run, we know for any $X \subseteq D_{2 k+1}-R_{2 k+1}$, if $S-\left(A_{k}^{\text {old }}+R_{2 k}^{\text {old }}\right)+X \in \mathcal{I}_{1}$, then $S-A_{k}^{\text {old }}+X \in \mathcal{I}_{1}$. That is, $S-\left(A_{k}+R_{2 k}\right)+R_{2 k}^{\text {old }}+X \in \mathcal{I}_{1}$. Our goal is to show that $S-A_{k}^{\text {old }}+A+X \in \mathcal{I}_{1}$.

Since $S-A_{k}^{\text {old }}+A+B_{k+1} \in \mathcal{I}_{1}$, we can find $\bar{A} \subseteq A$ and $\bar{B} \subseteq B_{k+1} \backslash X$ s.t. $S-A_{k}^{\text {old }}+\bar{A}+X+\bar{B} \in \mathcal{I}_{1}$ and $|\bar{A}|+|\bar{B}|=|A|+\left|B_{k+1}\right|-|X|$. Now if $\bar{A} \neq A$ then $|\bar{B}|>\left|B_{k+1}\right|-|X|$ and hence $X+\bar{B} \subseteq$ $D_{2 k+1}-R_{2 k+1}$ has size bigger than $\left|B_{k+1}\right|$. But this contradicts the fact that $B_{k+1} \subseteq D_{2 k+1}-R_{2 k+1}$ is maximally independent in $S-A_{k}^{\text {old }}$. Thus we must have $\bar{A}=A$ and $S-A_{k}^{\text {old }}+A+X \in \mathcal{I}_{1}$.

Lemma 42 (Properties of Refine2). Let $A_{k}^{\text {old }}, B_{k}^{\text {old }}$ be the $\left(A_{k}, B_{k}\right)$ before call to Refine2( $\left.k\right)$. After the call, all four invariants are preserved. Moreover, for $k \leq \ell$ a total of $\left|B_{k}^{\text {old }}\right|-\left|A_{k}^{\text {old }}\right|$ elements which were formerly fresh but are now selected, or were formerly selected but now removed.

Proof. After running Refine2( $k)$, we have $\left|A_{k}\right|=\left|B_{k}\right|$ by Lemma 40 . Also note that $\left|B_{k}^{\text {old }}\right|-\left|B_{k}\right|$ elements removed and $\left|A_{k}\right|-\left|A_{k}^{\text {old }}\right|$ selected for a total of $\left|B_{k}^{\text {old }}\right|-\left|A_{k}^{\text {old }}\right|$ elements changing status.

Invariants (a) and (c) remain true because $A_{k-1}$ is unchanged and $B_{k}$ only loses elements. For Invariant (b), observe that Refine2 simply finds a maximally independent subset of $S-\left(D_{2 k}-\right.$ $\left.R_{2 k}\right)+B_{k}^{\text {old }}+F_{2 k}^{\text {old }}=S-A_{k}^{\text {old }}+B_{k}^{\text {old }}$ so $\operatorname{rank}_{2}\left(S-A_{k}+B_{k}\right)$ remains unchanged. 
Invariant (d) remains true essentially by design. Refine2 finds a base of $S-\left(D_{2 k}-R_{2 k}\right)+$ $B_{k}^{\text {old }}$ and moves any of element not in the base into $R_{2 k-1}$. Those elements are in the span by definition.

Now by summing over all $k$ in the last two lemmas, we get the following corollary.

Corollary 43. After applying Refine, at least a total of $\left|B_{1}^{\text {old }}\right|-\left|B_{\ell+1}^{\text {old }}\right|$ elements are formerly fresh but now selected or formerly selected but now removed.

Proof. This essentially follows from the last two lemmas. Readers may notice that the sum does not exactly telescope because at the time Refine2 $(k)$ is applied, $B_{k}$ has been modified by Refine1 $(k)$. However this is okay as Refine1 $(k)$ only adds elements to the old $B_{k}$. Same for Refine2 $(k)$ which only adds elements to $A_{k+1}$ and can only help Refine1 $(k+1)$.

Using the properties established above, we show how Refine can be repeatedly used to find maximal augmenting sets.

Lemma 44. Suppose we run Refine until no more element changes its type. At this point, the collection $\Pi_{\ell}=\left(B_{1}, A_{1}, B_{2}, A_{2}, \cdots, A_{\ell}, B_{\ell+1}\right)$ is a maximal augmenting set.

Proof. For the sake of contradiction, assume there exists $\widetilde{\Pi}_{\ell}$ containing $\Pi_{\ell}$. Then by Theorem 33 and Theorem 34, there is a sequence of shortest augmenting paths in $G(S) \mid \Pi_{\ell}$. Taking the first path in the sequence, we have a shortest path $\left(b_{1}, a_{1}, b_{2}, a_{2}, \ldots, a_{\ell}, b_{\ell+1}\right)$ in $G(S) \mid \Pi_{\ell}$. In particular, $a_{k} \notin A_{k}$ and $b_{k} \notin B_{k}$ for any $k$.

We claim that all $a_{k}, b_{k}$ must have been removed by Refine. This would be a contradiction because an element in $D_{2 \ell+1}$ is removed only if it is in $\operatorname{span}_{2}\left(S+B_{\ell+1}\right)$. But in that case $b_{\ell+1}$ would not be in $D_{2 \ell+1}^{\prime}$ of $G^{\prime}=G(S) \mid \Pi_{\ell}$. We prove this claim by induction.

First, notice $b_{1}$ cannot be fresh. Otherwise $b_{1}$ would have been selected by Refine since $S+$ $B_{1}+b \in \mathcal{I}_{1}$. So $b_{1}$ must have been removed by Refine. Now we have two cases.

Suppose $b_{1}, a_{1}, \ldots, b_{k}$ have been removed. We need to show $a_{k}$ is removed. Suppose not, and suppose $a_{k} \in F_{2 k}$. Since $S-\left(A_{k}+a_{k}\right)+B_{k}+b_{k} \in \mathcal{I}_{2}, S-\left(D_{2 k}-R_{2 k}\right)+B_{k}+b_{k} \in \mathcal{I}_{2}$. But this is a contradiction as $b_{k} \in R_{2 k-1} \subseteq \operatorname{span}_{2}\left(S-D_{2 k}+R_{2 k}+B_{k}\right)$.

Now, suppose $b_{1}, a_{1}, \ldots, a_{k}$ have been removed. We need to show $b_{k+1}$ is removed. Suppose not, and suppose $b_{k+1} \in F_{2 k+1}$. Then $X=B_{k+1}+b_{k+1} \subseteq B_{k+1}+F_{2 k+1}$. Since $S-A_{k}-a_{k}+X \in \mathcal{I}_{1}$ and $a_{k} \in R_{2 k}, S-A_{k}-R_{2 k}+X \in \mathcal{I}_{1}$. Invariant (c) then implies $S-A_{k}+X \in \mathcal{I}_{1}$, which is a contradiction since $|X|=\left|A_{k}\right|+1$ and $X \in \operatorname{span}_{1}(S)$ (because $k+1>1$ ).

Lemma 45. Refine1 $(k)$ makes $O\left(\left|D_{2 k}\right|+\left|D_{2 k+1}\right|\right)$ independence oracle calls. Refine2 $(k)$ makes $O\left(\left|D_{2 k}\right|+\left|D_{2 k-1}\right|\right)$ independence oracle calls. Refine takes $O\left(n \cdot \mathcal{T}_{\text {ind }}\right)$-time.

Proof. Adding maximal $L \subseteq M$ to an independent set $N$ s.t. $N+L$ is still independent requires only adding elements of $L$ one at a time. This establishes the runtime of Refine1 and Refine2. Now Refine takes $\sum_{k} O\left(\left|D_{2 k}\right|+\left|D_{2 k+1}\right|\right)+O\left(\left|D_{2 k}\right|+\left|D_{2 k-1}\right|\right)=O(n)$ time since $n \geq \sum_{k}\left|D_{k}\right|$.

At this point, we can get an $O\left(n^{2} / \epsilon \cdot \mathcal{T}_{\text {ind }}\right)$-time algorithm. The algorithm runs in phases; in each phase the shortest augmenting path length of the exchange graph goes up by $\geq 2$. In each phase, we use Refine till we find the maximal augmenting set as given by Lemma 6.3. Since each element can change its type at most twice, we know that we need only $O(n)$ runs of Refine in each phase. By Lemma 45, Refine makes $O(n)$ independence oracle calls. Thus, each phase takes $O\left(n^{2}\right)$ independence oracles. Finally, to get an $(1-\epsilon)$-approximation, we need only $O(1 / \epsilon)$ phases, and thus the total time of this algorithm is a (not too impressive) $O\left(n^{2} / \epsilon \cdot \mathcal{T}_{\text {ind }}\right)$-time. We improve this with a hybrid approach in the next section. 


\subsection{Going Subquadratic by Combining Augmenting Sets and Augmenting Paths}

We obtain a faster algorithm by exploiting Lemma 39. The algorithm is parametrized by an integer $p$ which we set in the end. The algorithm runs in phases. At the beginning of a phase $\ell$, the algorithm first invokes Algorithm 4 to get the layers $D_{1}, D_{2}, \ldots, D_{2 \ell+1}$ in $O\left(n \log r \cdot \mathcal{T}_{\text {ind }}\right)$ time (Lemma 19). Throughout a phase $\ell$ if $S$ is the current solution, then the exchange graph $G(S)$ has shortest augmenting path length $2(\ell+1)$. After a phase, the shortest augmenting path length is $\geq 2(\ell+3)$. We run for $O(1 / \epsilon)$ phases.

In phase $\ell$, for $\ell \leq O(1 / \epsilon)$, we run the following routine.

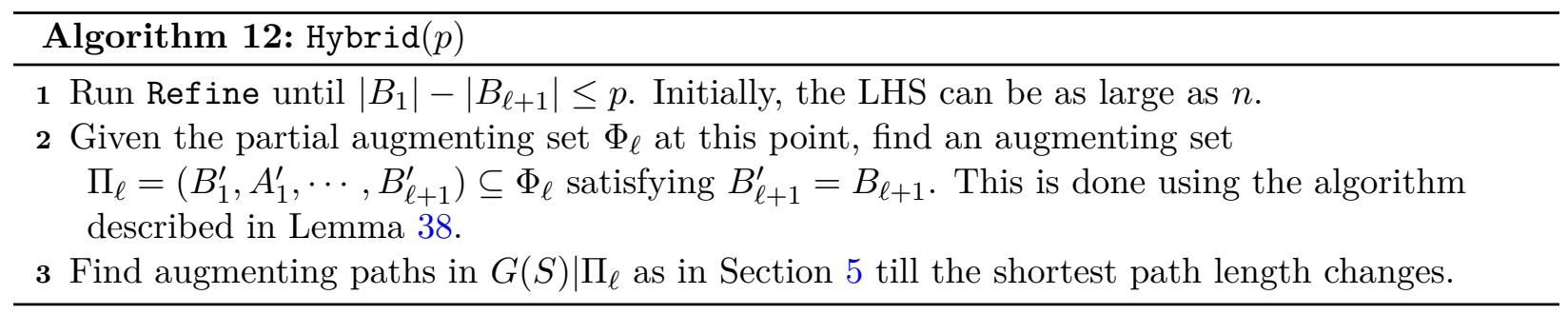

To analyze the running time of the hybrid algorithm, we go through the following steps. First, we show (Lemma 46) Line 1 takes $O\left(n^{2} / p\right)$-independence oracle calls. This is similar to the argument in the last paragraph of the previous section. Second, we show (Lemma 47) that $\Pi_{\ell}$ found in Line 2 is contained in a maximal augmenting set $\widetilde{\Pi}_{\ell}$ of width $\leq\left|B_{1}\right|$. Note that the width of $\Pi_{\ell}$ is $\left|B_{\ell+1}\right|$. Thus, the width of $\widetilde{\Pi}_{\ell} \backslash \Pi_{\ell}$ in $G(S) \mid \Pi_{\ell}$ is $\leq\left|B_{1}\right|-\left|B_{\ell+1}\right| \leq p$ (because of Line 1). Lemma 39 then implies any augmenting set in $G(S) \mid \Pi_{\ell}$ is of size $\leq(2 \ell+4) p$. Which means, in Line 3 we need to make at most these many augmentations. Since each augmentation takes $\tilde{O}(n)$ independence oracle calls, Line 3 will run in time $\tilde{O}\left(n p \ell \cdot \mathcal{T}_{\text {ind }}\right)$. By selecting $p \approx \sqrt{n}$, we get the desired result.

Lemma 46. Line 1 of the Hybrid algorithm takes $O\left(n^{2} / p \cdot \mathcal{T}_{\text {ind }}\right)$-time.

Proof. By Corollary 43, each call to Refine before Line 1 terminates changes the type of at least $\left|B_{1}\right|-\left|B_{\ell+1}\right|>p$ elements. Since there are $n$ elements and their type can change only twice, Refine can only be run for $O(n / p)$ times. By Lemma 45, each run of Refine makes $O(n)$ independence oracle calls. The lemma follows.

Lemma 47. Let $\Phi_{\ell}=\left(B_{1}, A_{1}, B_{2}, \ldots, B_{\ell+1}\right)$ be the partial augmenting sets obtained by running Refine for some number of times. Let $\Pi_{\ell} \subseteq \Phi_{\ell}$ be an augmenting set. Then, there is a maximal augmenting set $\widetilde{\Pi}_{\ell}$ containing $\Pi_{\ell}$ and the width of $\widetilde{\Pi}_{\ell}$ is at most $\left|B_{1}\right|$.

Proof. Given $\Pi_{\ell}$, we show a way of applying Refine to get a maximal augmenting set as in Lemma 44, such that no element in $\Pi_{\ell}$ is removed. Since $B_{1}$ was a maximal (this is why we run Refine1(0) in the end) set of elements that can be added while preserving independence, the maximal augmenting set found can't have width more than $\left|B_{1}\right|$. This will prove the lemma.

We now show how Refine1 and Refine2 can be implemented without removing any elements from $\Pi_{\ell}$. Let $\Pi_{\ell}=\left(B_{1}^{\prime}, A_{1}^{\prime}, \ldots, A_{\ell}^{\prime}, B_{\ell+1}^{\prime}\right)$.

Refine1 $(k)$ finds a maximal $A \subseteq A_{k}$ s.t. $S-A_{k}+B_{k+1}+A \in \mathcal{I}_{1}$. Since $S-A_{k}^{\prime}+B_{k+1}^{\prime} \in \mathcal{I}_{1}$, $A$ can be chosen so that $A \cap A_{k}^{\prime}=\emptyset$ and consequently no element of $A_{k}^{\prime}$ is removed.

Refine2(k) finds a maximally independent subset of $S-D_{2 k}+R_{2 k}+B_{k}+F_{2 k}=S-A_{k}+B_{k}$ by adding elements of $B_{k}$ first. Since $S-D_{2 k}+R_{2 k} \subseteq S-A_{k} \subseteq S-A_{k}^{\prime}$ and $S-A_{k}^{\prime}+B_{k}^{\prime} \in \mathcal{I}_{2}$, $B$ can be chosen so that $B_{k}^{\prime} \subseteq B$ and consequently no element of $B_{k}^{\prime}$ is removed. This finishes the proof of the lemma.

We now bound the running time of our algorithm. 
Theorem 48. Phase $\ell$ runs in $O\left(\left(n^{2} / p+n p \ell \log r\right) \cdot \mathcal{T}_{\text {ind }}\right)$ time plus the amortized cost $O\left(\sum_{a \in V}\left(d_{a}^{\text {end }}\right.\right.$ $\left.\left.d_{a}^{\text {start }}\right) \log r \cdot \mathcal{T}_{\text {ind }}\right)$, where $d_{a}^{\text {end }}$ and $d_{a}^{\text {start }}$ are the distances of a at the end and start of the phase respectively.

Proof. From Lemma 46, we get that Line 1 of the hybrid algorithm takes $O\left(n^{2} / p \cdot \mathcal{T}_{\text {ind }}\right)$ time. Let $\widetilde{\Pi}_{\ell}$ be the maximal augmenting set containing $\Pi_{\ell}$ as in Lemma 47. Line 2 , from Lemma 38 makes $O(n)$ independence oracle calls.

The width of $\widetilde{\Pi}_{\ell} \backslash \Pi_{\ell}$ is bounded by $\left|B_{1}\right|-\left|B_{\ell+1}\right| \leq p$. Now, consider $G(S) \mid \Pi_{\ell}$ and let $\mathcal{P}$ be the largest ordered collection of consecutive shortest augmenting paths. To find an augmenting path, we invoke Lemmas 19 and 20. Recall that Lemma 19 updates distances in $O\left(\sum_{a \in V}\left(1+d_{a}-\right.\right.$ $\left.\left.d_{a}^{\prime}\right) \log r \cdot \mathcal{T}_{\text {ind }}\right)$ time and, given distances, Lemma 20 finds an augmenting path in $O\left(n \cdot \mathcal{T}_{\text {ind }}\right)$ time. Since $d_{a}-d_{a}^{\prime}$ telescopes, in Line 3 computing $\mathcal{P}$ takes time

$$
O\left(\sum_{a \in V}\left(d_{a}^{\text {end }}-d_{a}^{\text {start }}\right) \log r \cdot \mathcal{T}_{\text {ind }}\right)+O\left(n|\mathcal{P}| \log r \cdot \mathcal{T}_{\text {ind }}\right) .
$$

Now, from Theorem 29, we have a maximal augmenting set $\Pi_{\ell}^{*}$ in $G(S) \mid \Pi_{\ell}$ with width $\left(\Pi_{\ell}^{*}\right)=|\mathcal{P}|$. From Lemma 39, we know width $\left(\Pi_{\ell}^{*}\right) \leq(2 \ell+4) \cdot \operatorname{width}\left(\widetilde{\Pi}_{\ell} \backslash \Pi_{\ell}\right)$. Thus, $|\mathcal{P}| \leq(2 \ell+4) \cdot\left(\left|B_{1}\right|-\left|B_{\ell+1}\right|\right) \leq$ $(2 \ell+4) p$. This proves the theorem.

We now have everything we need to prove our main theorem.

Proof of Theorem 21. Set $p=\lceil\sqrt{n \epsilon / \log r}\rceil$, and run the Hybrid algorithm until the shortest augmenting path length in $G(S) \geq 1 / \epsilon$. This requires at most $O(1 / \epsilon)$ phases. Moreover, when applying Lemma 19 we discard a vertex $a$ as soon as its distance (lower bound) is greater than $1 / \epsilon$. This is okay as distances are monotonic (Lemma 9) so any such vertex will not belong to any (future) shortest augmenting paths (or augmenting sets) which have length at most $1 / \epsilon$.

First we account for the total amortized cost arising from $O\left(\sum_{a \in V}\left(d_{a}^{\text {end }}-d_{a}^{\text {start }}\right) \log r \cdot \mathcal{T}_{\text {ind }}\right)$. By the above modification $d_{a}^{\text {end }} \leq 1 / \epsilon$ so they sum to at most $O\left(\frac{1}{\epsilon} n \log r \cdot \mathcal{T}_{\text {ind }}\right)$ which is dominated by our desired runtime.

At the beginning of each phase we take $O\left(n \log r \cdot \mathcal{T}_{\text {ind }}\right)$ time to construct the layers. Each phase, from the above Theorem, takes $O\left(n^{2} / p+n p \ell \log r\right)$ independence oracle calls. Thus the total time to run phase $\ell$ is $O\left(\left(n \log r+n^{2} / p+n p \ell \log r\right) \cdot \mathcal{T}_{\text {ind }}\right)=O\left(\left(n^{2} / p+n p \ell \log r\right) \cdot \mathcal{T}_{\text {ind }}\right)$ time. After all the phases are done, we are left with a set $S \in \mathcal{I}_{1} \cap \mathcal{I}_{2}$ such that the shortest augmenting path length in $G(S) \geq 1 / \epsilon$. This implies that $S$ is an $(1-\epsilon)$-approximate solution by Corollary 8 . The total running time is $O\left(\frac{1}{\epsilon} \cdot\left(\frac{n^{2}}{p}+n p \log r \frac{1}{\epsilon}\right) \cdot \mathcal{T}_{\text {ind }}\right)$. Since $p=\lceil\sqrt{n \epsilon / \log r}\rceil$, we prove the theorem.

\section{Approximately Optimal Fractional Solution via Frank-Wolfe}

Let $\mathcal{P}_{\mathcal{M}_{1}}, \mathcal{P}_{\mathcal{M}_{2}}$ be the matroid polytopes for matroids $\mathcal{M}_{1}=\left(V, \mathcal{I}_{1}\right)$ and $\mathcal{M}_{2}=\left(V, \mathcal{I}_{2}\right)$ respectively. Let $r$ be the size of their largest common independent set. It is well-known that the matroid intersection polytope is precisely $\mathcal{P}_{\mathcal{M}_{1}} \cap \mathcal{P}_{\mathcal{M}_{2}}$, i.e., $\mathcal{P}_{\mathcal{M}_{1}} \cap \mathcal{P}_{\mathcal{M}_{2}}$ is the convex hull of (the indicator vectors of) the common independent sets. The matroid intersection problem can therefore be solved via the following convex program:

$$
\max _{x \in \mathcal{P}_{\mathcal{M}_{1} \cap \mathcal{P}_{\mathcal{M}_{2}}}} 1^{\top} x
$$

In this section, we apply (a variant of) Frank-Wolfe to solve this problem faster: Theorem 50 obtain a $(1-\epsilon)$-optimal fractional solution $z$ (i.e., $\left.\sum_{i \in V} z_{i} \geq(1-\epsilon) \cdot r\right)$ in $O\left(n^{2} /\left(r \epsilon^{2}\right) \cdot \mathcal{T}_{\text {indep }}\right)$ time. 
To obtain an integral solution, we show in the next section that upon sampling via $x^{*}$ the size of the ground set can be reduced to $\tilde{O}\left(r / \epsilon^{2}\right)$. With this sparser new ground set we can speed up many matroid intersection algorithms. For instance, combining this with our previous $\tilde{O}\left(n^{1.5} / \epsilon^{1.5}\right)$ time algorithm would improve the runtime in the regime $r / \epsilon^{2} \leq n \leq r^{2} \epsilon$.

To find an approximate fractional solution efficiently, we relax the intersection constraint to

$$
\max _{x \in \mathcal{P}_{\mathcal{M}_{1}}, y \in \mathcal{P}_{\mathcal{M}_{2}}} 1^{\top} x-\frac{\eta}{2}\|x-y\|^{2},
$$

where $\eta$ is some parameter to be chosen later. This transformation is useful as it allows us to solve two disjoint greedy problems in an iteration of Frank-Wolfe. Our algorithm relies on the following theorem for constrained convex optimization.

Theorem 49 (Frank-Wolfe Algorithm [22, Theorem 1]). Given a convex set $K \subset \mathbb{R}^{n}$ and a convex function $f$ defined on $K$, suppose that:

- For any vector $c \in \mathbb{R}^{n}$, we can find $z_{c} \in \operatorname{argmax}_{z \in K} c^{\top} z$ in time $\mathcal{T}_{K}$.

- For any vector $z \in \mathbb{R}^{n}$, we can compute $\nabla f(z)$ in time $\mathcal{T}_{f}$.

- There is a constant $C_{f}$ such that for any $u, v \in K$ and any $\gamma \in[0,1]$, we have

$$
f(u+\gamma(v-u)) \leq f(u)+\langle\nabla f(u), \gamma(v-u)\rangle+\frac{\gamma^{2} C_{f}}{2} .
$$

In $k$ iterations, we can find $a z \in K$ such that

$$
f(z) \leq \min _{z^{\prime} \in K} f\left(z^{\prime}\right)+\frac{2 C_{f}}{k+2} .
$$

Each iteration takes $O\left(n+\mathcal{T}_{K}+\mathcal{T}_{f}\right)$.

For problem (7.1), we note that to optimize linear functions of $x$ and $y$ over $\left\{x \in \mathcal{P}_{\mathcal{M}_{1}}, y \in \mathcal{P}_{\mathcal{M}_{2}}\right\}$ we only need the standard greedy method, which can be implemented using $n$ independence oracle calls. We use this fact to prove the following main theorem of this section.

Theorem 50. Given two matroids $\mathcal{M}_{1}, \mathcal{M}_{2}$ via independence oracles, we can find a $z \in \mathcal{P}_{\mathcal{M}_{1}} \cap$ $\mathcal{P}_{\mathcal{M}_{2}}$ in $O\left(\frac{n}{r \epsilon^{2}}\right)$ iterations such that $\sum_{i \in V} z_{i} \geq(1-\epsilon) r$, where $r$ is the size of the largest common independent set and each iteration takes $O\left(n \cdot \mathcal{T}_{\text {ind }}\right)$ time.

Proof. Ideally, we want to apply Frank-Wolfe directly on the problem

$$
\max _{x \in \mathcal{P}_{\mathcal{M}_{1}}, y \in \mathcal{P}_{\mathcal{M}_{2}}} 1^{\top} x-\frac{\eta}{2}\|x-y\|_{2}^{2}
$$

with large $\eta$. However, the runtime of Frank-Wolfe algorithm depends implicitly on the diameter of the convex set via the constant $C_{f}$. To obtain a better $C_{f}$, we truncate both matroids by the size of largest common independent set $r$. Suppose for now, we know $\bar{r}$ such that $r \leq \bar{r} \leq 2 r$. We define the truncated set by

$$
K=\left\{(x, y) \in \mathcal{P}_{\mathcal{M}_{1}} \times \mathcal{P}_{\mathcal{M}_{2}}: \sum_{i} x_{i} \leq \bar{r}, \sum_{i} y_{i} \leq \bar{r}\right\}
$$


Now, we apply Frank-Wolfe on the function $f(x, y)=-1^{\top} x+\frac{\eta}{2}\|x-y\|_{2}^{2}$ over $K$. Theorem 49 shows that we can find some $x^{(k)}, y^{(k)}$ such that

$$
f\left(x^{(k)}, y^{(k)}\right) \leq \min _{(x, y) \in K} f(x, y)+\frac{2 C_{f}}{k+2} .
$$

We bound $C_{f}$ as follows:

$$
\begin{aligned}
C_{f} & =\sup _{u, v \in K, \gamma \in[0,1]} \frac{2}{\gamma^{2}}(f(u+\gamma(v-u))-f(u)-\langle\nabla f(u), \gamma(v-u)\rangle) \\
& =\sup _{u, v \in K, \gamma \in[0,1]} \frac{\eta}{\gamma^{2}}\|\gamma(v-u)\|_{2}^{2} \\
& \leq \sup _{u, v \in K} \eta\|v-u\|_{1} \leq 8 \eta r,
\end{aligned}
$$

where we used $K \subset[0,1]^{n}$ and $\sum_{i} u_{i}+\sum_{i} v_{i} \leq 2 \bar{r} \leq 4 r$. By considering the largest common independent set as a solution, we know $\min _{(x, y) \in K} f(x, y) \leq-r$. Hence, from the guarantee of Frank-Wolfe, we get

$$
-1^{\top} x^{(k)}+\frac{\eta}{2}\left\|x^{(k)}-y^{(k)}\right\|_{2}^{2} \leq-r+\frac{16 \eta r}{k+2} .
$$

To get a valid fractional solution, we define the vector $z$ by $z_{i}=\min \left\{x_{i}^{(k)}, y_{i}^{(k)}\right\}$. Since $x^{(k)} \in$ $\mathcal{P}_{\mathcal{M}_{1}}, y^{(k)} \in \mathcal{P}_{\mathcal{M}_{2}}$, we have that $z \in \mathcal{P}_{\mathcal{M}_{1}} \cap \mathcal{P}_{\mathcal{M}_{2}}$. Now, we bound the size of $z$ :

$$
\begin{aligned}
1^{\top} z & \geq 1^{\top} x^{(k)}-\left\|x^{(k)}-y^{(k)}\right\|_{1} \\
& \geq 1^{\top} x^{(k)}-\sqrt{n} \cdot\left\|x^{(k)}-y^{(k)}\right\|_{2} \\
& \geq 1^{\top} x^{(k)}-\frac{\eta}{2}\left\|x^{(k)}-y^{(k)}\right\|_{2}^{2}-\frac{n}{2 \eta} \\
& \geq r-\frac{16 \eta r}{k+2}-\frac{n}{2 \eta} .
\end{aligned}
$$

Picking $\eta=\sqrt{\frac{n(k+2)}{32 r}}$, we have

$$
1^{\top} z \geq r-\sqrt{\frac{32 n r}{k+2}} .
$$

Therefore, to get a fractional solution with an additive error of $\epsilon r$, we can set $k=\frac{32}{\epsilon^{2}} \frac{n}{r}$.

Finally, each iteration involves optimizing a linear function over $K$ which can be done by the greedy method using $O(n)$ independence oracle calls. To find $\bar{r}$, we can run the greedy algorithm to find a maximal common independent set in the two matroids using $O(n)$ independence oracles. Every maximal solution gives a 1/2-approximation to $r$ by Lemma 7 because it implies each augmenting path is of length at least 3 .

\section{Faster Algorithms by Sparsification}

From Section 7, we are given a point $x \in \mathcal{P}_{\mathcal{M}_{1}} \cap \mathcal{P}_{\mathcal{M}_{2}}$ in the matroid intersection polytope such that $\|x\|_{1} \geq(1-\epsilon) \cdot r$. In this section, we show how $x$ can be used to sparsify the matroids $\mathcal{M}_{1}$ and $\mathcal{M}_{2}$. In particular, we reduce the matroid intersection problem to solving it on a universe of size $O\left(\frac{r}{\epsilon^{2}} \log n\right)$. We can then use algorithms developed in the previous sections on this smaller universe to find an approximate integral solution. We use the following lemma of Karger (recall, $\operatorname{PackNum}(\mathcal{M})$ is the maximum number of disjoint bases in $\mathcal{M})$. 
Theorem 51 ([23, Theorem 4.1]). Given a matroid $\mathcal{M}$ with $\operatorname{PackNum}(\mathcal{M})=k$, suppose we sample each element of $\mathcal{M}$ with probability $p \geq 18 \ln n \cdot \frac{1}{k \epsilon^{2}}$, yielding a submatroid $\mathcal{M}(p)$ of $\mathcal{M}$. Then with high probability in $n$, we have $\operatorname{PackNum}(\mathcal{M}(p)) \in[(1-\epsilon) p k,(1+\epsilon) p k]$.

Our strategy to sparsify the matroids is to show that after appropriately sampling using $x$, the sampled elements $V^{\prime}$ will have $\left|V^{\prime}\right| \ll n$ and will form a universe $V^{\prime}$ which is almost "packed" for both the matroids, i.e., $V^{\prime}$ decomposes into an "almost perfect" partition of independent sets of size $(1-O(\epsilon)) r$ for both the matroids. Taking the average of these independent sets shows that the vector $\frac{r}{\left|V^{\prime}\right|} \cdot 1_{V^{\prime}}$ is almost independent for both the matroids. This means our sampling procedure is valid because $\left\|\frac{r}{\left|V^{\prime}\right|} \cdot 1_{V^{\prime}}\right\|_{1}=(1-O(\epsilon)) \cdot r$.

We first demonstrate how to sample using $x$ to obtain a new universe $V^{\prime}$ with the desired properties in a single matroid $\mathcal{M}$. This can be seen as a non-uniform version of Karger's result. Note that any matroid $\mathcal{M}$ naturally induces a new matroid if its elements are replaced by multiple identical copies (a set with multiple copies of an element is dependent).

Lemma 52. Suppose we are given a point $x \in \mathcal{P}_{\mathcal{M}}$ with $\|x\|_{1} \geq(1-\epsilon) \cdot r$. Set $\lambda=\frac{\epsilon r}{4 n^{3}}$. Suppose we replace each element $i \in V$ with $\frac{x_{i}^{\prime}}{\lambda}:=\left\lfloor\frac{x_{i}}{\lambda}\right\rfloor$ identical copies to form a new matroid $\mathcal{M}^{\prime}$ with universe $V^{\prime}$. Let submatroid $\mathcal{M}^{\prime}(p)$ on universe $V^{\prime}(p)$ be obtained by independently sampling each element of $V^{\prime}$ with probability $p=O\left(\frac{\lambda \ln (n / \epsilon)}{\epsilon^{2}}\right)$. Then with high probability in $n$,

(a) $V^{\prime}(p)$ has size at most $(1-O(\epsilon)) \cdot r p / \lambda=O\left(\frac{r}{\epsilon^{2}} \ln \frac{n}{\epsilon}\right)$.

(b) $V^{\prime}(p)$ contains $(1-O(\epsilon)) \cdot\left|V^{\prime}(p)\right| / r$ disjoint independent sets of size $(1-O(\epsilon)) r$.

Proof. Note that $x^{\prime} \in \mathcal{P}_{\mathcal{M}}$ is a multiple of $\lambda$ and $\left\|x^{\prime}\right\|_{1}=(1-O(\epsilon)) \cdot r$ as the total loss of rounding is at most $n \lambda$.

For (1), the expected size of $V^{\prime}(p)$ is

$$
\sum_{i} \frac{x_{i}^{\prime}}{\lambda} \cdot p=(1-O(\epsilon)) \cdot r p / \lambda=O\left(\frac{r}{\epsilon^{2}} \ln \frac{n}{\epsilon}\right),
$$

which holds w.h.p. within a factor of $1 \pm \epsilon$ by standard Chernoff bound.

For (2), we further slightly decrease $x^{\prime}$ to obtain a vector $\bar{x} \in \mathcal{P}_{\mathcal{M}}$ so that $\|\bar{x}\|_{1}=\left\lfloor\left\|x^{\prime}\right\|_{1}\right\rfloor:=\bar{r}$. Clearly $\bar{r}=(1-O(\epsilon)) \cdot r$. Now by the Carathï¿œodory's theorem and the integrality of the matroid (base) polytope, $\bar{x}$ can be written as a convex combination of size- $\bar{r}$ independent sets, ${ }^{5}$ i.e., $\bar{x}=\sum_{i=1}^{n} t_{i} v_{i}$ where $v_{i}$ are indicator vectors of some size $-\bar{r}$ independent sets and multipliers $t_{i} \geq 0$ satisfy $\sum_{i} t_{i}=1$.

We round $t$ down to $t^{\prime}$ so that it is a multiple of $\lambda$. Now by considering $t^{\prime} / \lambda$ copies of $v_{i}$ for all $i$, we see that $V^{\prime}$ contains at least

$$
\sum_{i} \frac{t_{i}^{\prime}}{\lambda} \geq \frac{1}{\lambda}-n=\left(1-\frac{r \epsilon}{4 n^{2}}\right) \frac{1}{\lambda}=(1-o(\epsilon)) \frac{1}{\lambda}
$$

disjoint size- $\bar{r}$ independent sets. Moreover, the size of set is $\left|V^{\prime}\right|=\sum_{i} \frac{x_{i}^{\prime}}{\lambda}=(1-O(\epsilon)) \cdot \frac{r}{\lambda}$, which means $\ln \left|V^{\prime}\right|=O(\ln (n / \epsilon))$. So by Theorem 51, w.h.p. $\mathcal{M}^{\prime}(p)$ contains at least

$$
(1-\epsilon) p \cdot(1-o(\epsilon)) \frac{1}{\lambda}=(1-O(\epsilon)) \cdot \frac{\left|V^{\prime}(p)\right|}{r}
$$

disjoint size- $\bar{r}$ independent sets, as desired.

\footnotetext{
${ }^{5}$ This can be seen as follows: truncate $\mathcal{M}$ to size $\bar{r}$. Then any fractional independent set of size $\bar{r}$ is a convex combination of independent sets, which are of size at most $\bar{r}$ and hence exactly $\bar{r}$.
} 
From (2) of Lemma 52, the universe $V^{\prime}(p)$ almost can be partitioned into disjoint independent sets of size $(1-O(\epsilon)) \cdot r$. We now show that $V^{\prime}(p)$ has a common independent set of size $(1-O(\epsilon)) \cdot r$ by putting equal mass on almost every element.

Lemma 53. For a given point $x \in \mathcal{P}_{\mathcal{M}_{1}} \cap \mathcal{P}_{\mathcal{M}_{2}}$ with $\|x\|_{1} \geq(1-\epsilon) \cdot r$, let $V^{\prime}(p)$ be sampled as in Lemma 52. Then $V^{\prime}(p)$ has a common independent set of size $(1-O(\epsilon)) \cdot r$.

Proof. Suppose $V^{\prime}(p)$ contains disjoint $I_{1}^{(1)}, \ldots, I_{k_{1}}^{(1)} \in \mathcal{I}_{1}$ and disjoint $I_{1}^{(2)}, \ldots, I_{k_{2}}^{(2)} \in \mathcal{I}_{2}$ all of which are of size $(1-O(\epsilon)) \cdot r$. By Lemma 52 , we may take $k_{1}, k_{2}=(1-O(\epsilon)) \cdot\left|V^{\prime}(p)\right| / r$.

We begin with a few simplifications. Let $k=\min \left\{k_{1}, k_{2}\right\}$ and WLOG assume that all $I_{\alpha}^{(1)}$ and $I_{\beta}^{(2)}$ are of equal size $r^{\prime}=(1-O(\epsilon)) \cdot r$ by dropping elements from the non-smallest independent sets. For $i=1,2$, define $y^{(i)} \in \mathbb{R}^{V^{\prime}(p)}$ by

$$
y_{a}^{(i)}= \begin{cases}1 / k & \text { if } a \in I_{1}^{(i)} \cup \ldots \cup I_{k}^{(i)} \\ 0 & \text { o.w. }\end{cases}
$$

Then $y^{(i)}$, which is the average of the indicator vectors for $I_{1}^{(i)}, \ldots, I_{k}^{(i)}$, belongs to the matroid polytope for $\mathcal{M}_{i}^{\prime}(p)$. Therefore $y=\min \left\{y^{(1)}, y^{(2)}\right\}$ belongs to the matroid intersection polytope for $\mathcal{M}_{1}^{\prime}(p), \mathcal{M}_{2}^{\prime}(p)$. Since the matroid intersection polytope is integral, it suffices to show that $y$ has size $(1-O(\epsilon)) \cdot r$.

We bound the size of $y$ as follows:

$$
\begin{aligned}
\|y\|_{1} & \geq\left\|y^{(1)}\right\|_{1}+\left\|y^{(2)}\right\|_{1}-\left|V^{\prime}(p)\right| / k \\
& =2 r^{\prime}-r /(1-O(\epsilon)) \\
& =2(1-O(\epsilon)) r-(1+O(\epsilon)) r \quad=\quad(1-O(\epsilon)) r,
\end{aligned}
$$

which completes the proof of this lemma.

Combining the last two lemmas shows that our sampling scheme reduces the size of the universe to $O\left(\frac{r}{\epsilon^{2}} \ln \frac{n}{\epsilon}\right)$.

Theorem 54. Given a point $x \in \mathcal{P}_{\mathcal{M}_{1}} \cap \mathcal{P}_{\mathcal{M}_{2}}$ such that $\|x\|_{1} \geq(1-\epsilon) \cdot r$, we can find in $O(n)$ time a set $V^{\prime}$ of size $O\left(\frac{r}{\epsilon^{2}} \log n\right)$ such that the largest common independent subset of $V^{\prime}$ for $\mathcal{M}_{1}$ and $\mathcal{M}_{2}$ has at least $(1-O(\epsilon)) \cdot r$ elements.

Proof. From Lemmas 52 and $53, V^{\prime}(p)$ satisfies all the desired properties. It remains to analyze the runtime of the sampling scheme. Recall that $V^{\prime}(p)$ is obtained by sampling $V^{\prime}$ which contains identical copies of the elements of $V$. Instead of creating and sampling $V^{\prime}$ explicitly, we can equivalently sample $i \in V$ as a binomial random variable with $\left\lfloor\frac{x_{i}}{\lambda}\right\rfloor$ trials and probability $p$.

Theorem 55. There is an $\tilde{O}\left(\left(\frac{n^{2}}{r \epsilon^{2}}+\frac{r^{1.5}}{\epsilon^{4.5}}\right) \cdot \mathcal{T}_{\text {ind }}\right)$-time algorithm to obtain a $(1-\epsilon)$-approximation to the matroid intersection problem.

Proof. Using Theorem 50, in $O\left(\left(n^{2} / r \epsilon^{2}\right) \cdot \mathcal{T}_{\text {ind }}\right)$ time we can find a point $z \in \mathcal{P}_{\mathcal{M}_{1}} \cap \mathcal{P}_{\mathcal{M}_{2}}$ with $\|z\|_{1} \geq(1-\epsilon) r$. Applying Theorem 54 on $z$, in $O(n)$ time we can reduce the ground set to $V^{\prime}$ with $\left|V^{\prime}\right|=O\left(r \log n / \epsilon^{2}\right)=: n^{\prime}$ and the maximum common independent set in $V^{\prime}$ has size $\geq(1-O(\epsilon)) r$. Note that any independence oracle query on the matroid restricted to $V^{\prime}$ doesn't change, and in particular takes $\mathcal{T}_{\text {ind }}$ time. Applying Theorem 21 on this universe of size $n^{\prime}$, we see that in $O\left(\left(n^{1.5} / \epsilon^{1.5}\right) \cdot \mathcal{T}_{\text {ind }}\right)$ time we can get a common independent set $S$ with $|S| \geq(1-\epsilon)\left|S^{*}\right|$ where $S^{*}$ is the largest common independent set in $V^{\prime}$.Thus, $|S| \geq(1-\epsilon) \cdot(1-O(\epsilon)) r=(1-O(\epsilon)) r$. That is, $S$ is an $(1-O(\epsilon))$-approximate solution. The time complexity follows by setting $n^{\prime}=O\left(r \log n / \epsilon^{2}\right)$ in the above. 


\section{Acknowledgment}

We are thankful to Kent Quanrud for pointing us to Karger's paper [23]. We are also thankful to Troy Lee for pointing us to [32], and for his comments on a previous version.

\section{References}

[1] Martin Aigner and Thomas A Dowling. Matching theory for combinatorial geometries. Transactions of the American Mathematical Society, 158(1):231-245, 1971.

[2] Carl Brezovec, Gerard Cornuéjols, and Fred Glover. Two algorithms for weighted matroid intersection. Math. Programming, 36(1):39-53, 1986.

[3] Chandra Chekuri and Kent Quanrud. Fast approximations for matroid intersection. In Proceedings, ACM-SIAM Symposium on Discrete Algorithms (SODA), 2016.

[4] Ho Yee Cheung, Lap Chi Lau, and Kai Man Leung. Algebraic algorithms for linear matroid parity problems. ACM Trans. on Algorithms (TALG), 10(3):10, 2014.

[5] Paul Christiano, Jonathan A Kelner, Aleksander Mądry, Daniel A Spielman, and Shang-Hua Teng. Electrical flows, laplacian systems, and faster approximation of maximum flow in undirected graphs. In Proceedings, ACM Symposium on Theory of Computing (STOC), pages 273-282. ACM, 2011.

[6] William H. Cunningham. Improved bounds for matroid partition and intersection algorithms. SIAM Journal on Computing (SICOMP), 15(4):948-957, 1986.

[7] Randall Dougherty, Chris Freiling, and Kenneth Zeger. Networks, matroids, and non-shannon information inequalities. IEEE Transactions on Information Theory, 53(6):1949-1969, 2007.

[8] Randall Dougherty, Chris Freiling, and Kenneth Zeger. Network coding and matroid theory. Proceedings of the IEEE, 99(3):388-405, 2011.

[9] Jack Edmonds. Submodular functions, matroids, and certain polyhedra. Combinatorial structures and their applications, pages 69-87, 1970.

[10] S. El Rouayheb, A. Sprintson, and C. Georghiades. On the index coding problem and its relation to network coding and matroid theory. IEEE Transactions on Information Theory, 56(7):3187-3195, 2010.

[11] Huy L. Nguy ên. A note on Cunningham's algorithm for matroid intersection. CoRR, abs/1904.04129, 2019.

[12] András Frank. A weighted matroid intersection algorithm. J. Algorithms, 2(4):328-336, 1981.

[13] Harold N. Gabow and Ying Xu. Efficient algorithms for independent assignment on graphic and linear matroids. In Proceedings, IEEE Symposium on Foundations of Computer Science (FOCS), pages 106$111,1989$.

[14] Harold N. Gabow and Ying Xu. Efficient theoretic and practical algorithms for linear matroid intersection problems. J. Comput. System Sci., 53(1):129-147, 1996.

[15] Guru Prashanth Guruganesh and Sahil Singla. Online matroid intersection: Beating half for random arrival. In Proceedings, MPS Conference on Integer Programming and Combinatorial Optimization (IPCO), pages 241-253. Springer, 2017.

[16] Nicholas J. A. Harvey. Matroid intersection, pointer chasing, and young's seminormal representation of $S_{n}$. In Proceedings, ACM-SIAM Symposium on Discrete Algorithms (SODA), pages 542-549, 2008.

[17] Nicholas J. A. Harvey. Algebraic algorithms for matching and matroid problems. SIAM Journal on Computing (SICOMP), 39(2):679-702, 2009.

[18] Nicholas J. A. Harvey. Query lower bounds for matroid intersection (combinatorial optimization and discrete algorithms). RIMS Kokyuroku Bessatsu, pages 81 - 105, 2010. 
[19] Julian Haselmayr. Schnitt von matroiden theorie und algorithmen. Master's thesis, University of Augsberg, 2008.

[20] John E. Hopcroft and Richard M. Karp. An $n^{5 / 2}$ algorithm for maximum matchings in bipartite graphs. SIAM Journal on Computing (SICOMP), 2(4):225-231, 1973.

[21] Chien-Chung Huang, Naonori Kakimura, and Naoyuki Kamiyama. Exact and approximation algorithms for weighted matroid intersection. In Proceedings, ACM-SIAM Symposium on Discrete Algorithms (SODA), pages 430-444, 2016.

[22] Martin Jaggi. Revisiting Frank-Wolfe: Projection-free sparse convex optimization. In Proceedings, International Conference on Machine Learning (ICML), volume 28, pages 427-435, 2013.

[23] David R. Karger. Random sampling and greedy sparsification for matroid optimization problems. Math. Programming, 82(1-2):41-81, 1998.

[24] Jonathan A Kelner, Yin Tat Lee, Lorenzo Orecchia, and Aaron Sidford. An almost-linear-time algorithm for approximate max flow in undirected graphs, and its multicommodity generalizations. In Proceedings, ACM-SIAM Symposium on Discrete Algorithms (SODA), pages 217-226, 2014.

[25] Eugene L. Lawler. Matroid intersection algorithms. Math. Programming, 9(1):31-56, 1975.

[26] Yin Tat Lee, Satish Rao, and Nikhil Srivastava. A new approach to computing maximum flows using electrical flows. In Proceedings, ACM Symposium on Theory of Computing (STOC), pages 755-764, 2013.

[27] Yin Tat Lee, Aaron Sidford, and Sam Chiu-wai Wong. A faster cutting plane method and its implications for combinatorial and convex optimization. In Proceedings, IEEE Symposium on Foundations of Computer Science (FOCS), pages 1049-1065. IEEE, 2015.

[28] Aleksander Mądry. Navigating central path with electrical flows: From flows to matchings, and back. In Proceedings, IEEE Symposium on Foundations of Computer Science (FOCS), pages 253-262, 2013.

[29] Marcin Mucha and Piotr Sankowski. Maximum matchings via gaussian elimination. In Proceedings, IEEE Symposium on Foundations of Computer Science (FOCS), pages 248-255, 2004.

[30] Kazuo Murota. Matrices and matroids for systems analysis, volume 20. Springer Science \& Business Media, 2009.

[31] Richard Peng. Approximate undirected maximum flows in o (m polylog (n)) time. In Proceedings, ACM-SIAM Symposium on Discrete Algorithms (SODA), pages 1862-1867, 2016.

[32] Christopher Price. Combinatorial algorithms for submodular function minimization and related problems. Master's thesis, University of Waterloo, 2015.

[33] András Recski. Matroid theory and its applications in electric network theory and in statics, volume 6. Springer Science \& Business Media, 2013.

[34] Jonah Sherman. Nearly maximum flows in nearly linear time. In Proceedings, IEEE Symposium on Foundations of Computer Science (FOCS), pages 263-269, 2013.

[35] Jonah Sherman. Area-convexity, $\ell_{\infty}$ regularization, and undirected multicommodity flow. In Proceedings, ACM Symposium on Theory of Computing (STOC), pages 452-460, 2017.

[36] Maiko Shigeno and Satoru Iwata. A dual approximation approach to weighted matroid intersection. Operations research letters, 18(3):153-156, 1995.

[37] Aaron Sidford and Kevin Tian. Coordinate methods for accelerating $\ell_{\infty}$ regression and faster approximate maximum flow. In Proceedings, IEEE Symposium on Foundations of Computer Science (FOCS), pages $922-933,2018$. 